DEPARTMENT OF ENVIRONMENT,

TECHNOLOGY AND TECHNOLOGY MANAGEMENT

\title{
Optimal Blocking for General Resolution-3 Designs
}

Eric D. Schoen, Bagus Sartono \& Peter Goos

\author{
UNIVERSITY OF ANTWERP \\ Faculty of Applied Economics \\ Stadscampus \\ Prinsstraat 13, B.226 \\ BE-2000 Antwerpen \\ Tel. +32(0)32654032 \\ Fax $+32(0) 32654799$ \\ http://www.ua.ac.be/tew
}




\title{
FACULTY OF APPLIED ECONOMICS
}

\author{
DEPARTMENT OF ENVIRONMENT, \\ TECHNOLOGY AND TECHNOLOGY MANAGEMENT
}

\section{Optimal Blocking for General Resolution-3 Designs}

Eric D. Schoen, Bagus Sartono \& Peter Goos

RESEARCH PAPER 2012-025

NOVEMBER 2012
University of Antwerp, City Campus, Prinsstraat 13, B-2000 Antwerp, Belgium Research Administration - room B.226 phone: (32) 32654032 fax: (32) 32654799
e-mail: joeri.nys@ua.ac.be

The papers can be also found at our website: www.ua.ac.be/tew (research $>$ working papers) \& www.repec.org/ (Research papers in economics - REPEC)

$D / 2012 / 1169 / 025$ 


\title{
Optimal Blocking for General Resolution-3 Designs
}

\author{
Eric D. Schoen ${ }^{1,2}$, Bagus Sartono ${ }^{1}$, and Peter Goos ${ }^{1,3}$ \\ ${ }^{1}$ University of Antwerp, Belgium \\ ${ }^{2}$ TNO, Research Group Quality and Safety, Zeist, Netherlands \\ ${ }^{3}$ Erasmus University Rotterdam, Netherlands
}

June 30, 2012

\begin{abstract}
This paper presents a general method for finding optimal blocking arrangements of pure-level and mixedlevel orthogonal designs of resolution 3. The method requires enumeration of all non-isomorphic 'parent designs' that include the treatment factors as well as the future blocking factor. For all these designs, word-length frequency vectors expressing the aliasing between main effects and two-factor interactions as well as the aliasing among two-factor interactions are calculated. The same is done for all projections into 'child designs' obtained by dropping any possible blocking factor. The word-length frequency vectors of the parent and child designs then allow the selection of blocking arrangements that are optimal with respect to five criteria appropriate for blocking resolution-3 orthogonal designs. We provide optimal blocking arrangements for orthogonal pure-level and mixed-level designs of 12, 16, 18, 20 and 27 runs.
\end{abstract}

KEY WORDS: Aliasing; Confounding; Generalized Word-Length Pattern; Orthogonal Array; Strength 2

\section{Introduction}

Effect estimates from a series of experimental runs can be sensitive to heterogeneous operating conditions. For example, chemical experiments are generally conducted with a limited number of reactors, where each of the reactors is used for a subset of the experimental runs. The challenge then is to group the runs so that uncontrollable differences between the reactors do not affect the estimates of the effects of the treatment factors. We call the groups of experimental runs blocks and the allocation of the runs to the blocks blocking. In this article, we focus on blocking pure-level and mixed-level orthogonal designs of resolution 3 or strength 2. 
Early work on blocking designs for industrial experiments was carried out in the late 1950s. This resulted in blocking arrangements of regular two-level designs produced by the National Bureau of Standards (1957), and of regular three-level designs listed by Connor and Zelen (1959). Later work concentrated on defining optimality criteria for blocking (Bisgaard, 1994; Sun et al., 1997; Sitter et al., 1997; Cheng and Wu, 2002). These criteria are based on the defining contrast subgroup, a mathematical construct that only exists for the sub-class of regular fractional factorial designs. Criteria for blocking two-level nonregular designs, which generalize those for regular two-level designs, were proposed by Cheng et al. (2004).

Finding blocking arrangements for nonregular three-level designs and mixed-level designs requires new methodology. The same is true for all two-level designs with a number of blocks other than a power of two. In this paper, we propose five different criteria for selecting blocking arrangements for general fractional factorial designs of resolution 3 , as well as a method for finding blocking arrangements that are optimal with respect to these criteria. Two of the five blocking criteria we use are new, while the remaining three are adaptations of existing criteria in the literature. The blocking criteria we propose are based on the assumption that a blocking factor does not interact with the treatment factors, while treatment factors may or may not interact with other treatment factors.

Like Cheng et al. (2004), we start from an exhaustive enumeration of designs. However, unlike the method of Cheng et al. (2004), which can only be used to find arrangements of two-level designs in a number of blocks that is a power of two, our approach permits the blocking of general factorial designs of resolution 3 in any number of blocks that is compatible with orthogonal blocking. For instance, our approach can be used to arrange 20-run two-level orthogonal designs in five blocks of four runs, to arrange a 12-run two-level orthogonal design in three blocks of four runs, and to arrange a 27-run design in nine blocks of three runs.

To illustrate our blocking approach, consider the design of a 27-run chemical experiment with eight three-level treatment factors and three blocks of nine runs. Such a design is suitable when three reactors are employed for the experiment. In total, the experiment has nine three-level factors, eight of which are treatment factors and one of which is the blocking factor. We call the design for the treatment factors a 'child design' and the full design, involving the treatment factors and the blocking factor, the 'parent design'.

Three parent designs are displayed in Table 1 . The levels of the treatment factors are represented by 0,1 , and 2, whereas the three levels of the blocking factor are labeled 'Reactor 1', 'Reactor 2' and 'Reactor 3'. In each of the designs, the treatment factors are orthogonal to each other and to the blocking factor. Because of the orthogonality of the treatment factors to the blocking factor, the estimates of the main effects of the treatment factors are not affected by block differences.

The three parent designs in Table 1 are nonregular orthogonal designs with nine three-level factors and 27 runs. Schoen et al. (2010) showed that there are 9793 different designs of this type. The blocking 
Table 1: Three optimally blocked $3^{8}$ designs in 3 blocks of size 9

\begin{tabular}{|c|c|c|c|}
\hline ID & Reactor 1 & Reactor 2 & Reactor 3 \\
\hline \multirow[t]{9}{*}{1} & $\begin{array}{lllllllll}0 & 0 & 0 & 0 & 0 & 0 & 0 & 0\end{array}$ & 01012021 & 02011202 \\
\hline & $\begin{array}{llllllllll}0 & 0 & 1 & 1 & 1 & 1 & 1 & 1\end{array}$ & $\begin{array}{llllllll}0 & 1 & 1 & 0 & 0 & 2 & 1 & 2\end{array}$ & 22010 \\
\hline & 002222222 & $\begin{array}{llllllllll}0 & 1 & 2 & 1 & 1 & 0 & 0\end{array}$ & 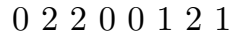 \\
\hline & 110011222 & 1220101010 & $\begin{array}{lllllll}1 & 0 & 0 & 2 & 2 & 1 & 0\end{array}$ \\
\hline & 11112200 & 12121221 & 101100022 \\
\hline & 11220011 & 12202002 & 10201210 \\
\hline & 2200022211 & 2000210012 & 21020220 \\
\hline & 221200102 & 20102 & 2111011001 \\
\hline & 22211020 & 20221020201 & 21212112 \\
\hline \multirow[t]{9}{*}{2} & 00 & 22 & 20 \\
\hline & $\begin{array}{llll}0 & 0 & 0 & 1\end{array}$ & 022 & 01 \\
\hline & 01100112 & 02212010 & 02221102 \\
\hline & 11202022 & 10101202 & 10110021 \\
\hline & 11221211 & 10122110 & 12001120 \\
\hline & 12012201 & 11210100 & 20012 \\
\hline & 20221020 & 1002 & 20202101 \\
\hline & 22112122 & 21020121 & 20210212 \\
\hline & 22120200 & 2210101011 & 2210 \\
\hline \multirow[t]{9}{*}{3} & 0000000000 & 21 & 0 \\
\hline & 011111 & 011 & 02102211 \\
\hline & 01100122 & 02012022 & 02211000 \\
\hline & 10112202 & 10200011 & 10201122 \\
\hline & 11222010 & 11010220 & 11111021 \\
\hline & 12021212 & 12102100 & 12020101 \\
\hline & 21212101 & 201210002 & 2000221200 \\
\hline & 22120021 & 21001201 & 20110210 \\
\hline & 2222012220 & 22221010112 & 2100022012 \\
\hline
\end{tabular}

arrangements presented in the table are optimal according to the different criteria that we utilize in this paper to select optimally blocked designs.

The remainder of this paper is organized as follows. In Section 2, we review the optimality criteria for completely randomized designs on which our criteria for blocking are based. We introduce our optimality criteria for blocking in Section 3. In Section 4, we describe how we can use different ways of sorting completely randomized designs for the purpose of blocking. We apply that approach in Section 5 to generate the best blocking arrangements for mixed-level and pure-level designs with 12, 16, 18, 20, and 27 runs, and describe the results. We conclude the paper with a brief discussion of the limitations of our approach, and two technical appendices in which we compare some of our results for 16 and 27 runs to those of Cheng et al. (2004) and Xu et al. (2004), respectively. 


\section{Criteria for completely randomized designs}

The goal of this paper is to find optimal blocking arrangements of general resolution-3 designs with $N$ runs and $k$ treatment factors. Hedayat et al. (1999) show that these designs are orthogonal arrays of strength 2. Formally, an orthogonal array is a rectangular arrangement of symbols in $N$ rows and $k$ columns, with $s_{j}$ different symbols in its $j$ th column. An array of strength 2 has the property that, for all pairs of columns, all pairs of symbols occur equally often (Rao, 1947). We denote the class of strength-2 arrays with $N$ rows, $k$ columns and $s_{j}$ levels for the $j$ th column by $\mathrm{OA}\left(N ; s_{1} \ldots s_{k}\right)$.

$\mathrm{Xu}$ and $\mathrm{Wu}$ (2001) propose the generalized word-length pattern (GWLP) to classify orthogonal arrays of any strength, number of factors, and numbers of factor levels. The GWLP of a design with $k$ factors and strength $t \geq 2$ is a vector $\left(A_{3}, A_{4}, A_{5}, \ldots, A_{k}\right)$. We call an element $A_{i}$ of the GWLP the generalized word count of length $i$. It is calculated as follows. First, for each factor $j$ in the design, $s_{j}-1$ orthogonal main-

effect contrast vectors with length $\sqrt{N}$ are constructed. Second, the element-wise products are calculated of all sets of $i$ main-effect contrast vectors corresponding to $i$ different main effects. Third, for each set of $i$ contrasts vectors, the element-wise products are summed and the resulting sum is squared. Finally, the squared sums corresponding to all sets are summed and divided by $N^{2}$.

The $A_{3}$ value quantifies the extent to which main-effect contrast vectors are aliased with two-factor interaction contrast vectors, and each other $A_{i}$ value, where $i>3$, measures the extent to which main-effect contrast vectors are aliased with $(i-1)$-factor interaction contrast vectors and $q$-factor interaction contrast vectors are aliased with $(i-q)$-factor interaction contrast vectors (for $q$ ranging from 2 to $i-2$ ). Both the word-length pattern for regular prime-level designs (Box et al., 2005; Wu and Hamada, 2009) and the extended word-length pattern for nonregular two-level designs (Tang and Deng, 1999; Deng and Tang, 1999) are special cases of the GWLP.

The resolution of a design is defined as the smallest $p$ for which $A_{p}>0$. All the designs discussed in this paper have at least resolution 3. To distinguish between designs of equal resolution, Xu and Wu (2001) use a generalized aberration (GA) criterion similar to the aberration criterion for regular fractional factorial two-level designs (Fries and Hunter, 1980). A minimum GA design sequentially minimizes the entries of the GWLP from left to right.

For the designs studied in this paper, aliasing of main-effect contrast vectors with two-factor interaction contrast vectors and among two-factor interaction contrast vectors are key issues. This is because, usually, the interest in industrial experimentation is in main effects and two-factor interactions, while higher-order interaction effects are considered negligible. We therefore restrict our attention to the first two elements of the GWLP, $A_{3}$ and $A_{4}$. As an illustration, the $A_{3}$ and $A_{4}$ values of the three eight-factor child designs in 
Table 2: Properties of the eight-factor child designs in Table 1. The elements of the $F A_{3}^{c}$ vector correspond to $A_{3}$ values of $2,2 / 3,14 / 27,4 / 9,8 / 27$, and 0 .

\begin{tabular}{clllrrrrr}
\hline ID & $A_{3}^{c}$ & $A_{4}^{c}$ & \multicolumn{6}{c}{$F A_{3}^{c}$} \\
\hline 1 & 16 & 60 & 2 & 18 & 0 & 0 & 0 & 36 \\
2 & 16 & 60 & 2 & 18 & 0 & 0 & 0 & 36 \\
3 & 20.30 & 45.85 & 0 & 1 & 11 & 16 & 23 & 5 \\
\hline
\end{tabular}

Table 1 are shown in the second and third column of Table 2 . We call these values $A_{3}^{c}$ and $A_{4}^{c}$ values, where the superscript ' $c$ ' is short for child design.

The eight-factor child designs from parent designs 1 and 2 have minimum GA. Each one of the two child designs can be obtained from the other by switching columns and relabeling levels. Therefore, the two child designs are isomorphic. They outperform the eight-factor child design obtained from parent design 3 in terms of the GA criterion because they have a smaller $A_{3}^{c}$ value.

Designs of resolution 3 are very often used for screening purposes, in which case only a few of the $k$ treatment factors are expected to be active. Therefore, the statistical properties of low-order projections of the $k$-factor designs are of utmost importance. The lower the order of the projections, the more important their properties for screening purposes. Since all projections of any orthogonal design into one factor result in an equireplicated one-factor design and all projections of any resolution-3 design into two factors result in at least one replicate of a full factorial design, projections into one and two factors cannot be used to distinguish between different designs of resolution 3. Therefore, the most important projection is the projection into three factors.

To quantify the projection properties of a design into three factors, Xu et al. (2004) propose to determine the $A_{3}$ value of the projections. If there are $k$ factors in total, then the number of projections into three factors equals $k(k-1)(k-2) / 6$. Each of these projections yields a certain $A_{3}$ value. Usually, there are only a limited number of different $A_{3}$ values. We call the vector of frequencies with which each of these $A_{3}$ values occurs in a three-factor projection $F A_{3}$. This vector is a generalization of the confounding frequency vector introduced by Deng and Tang (1999) for nonregular two-level designs.

The $F A_{3}$ vectors of the three eight-factor child designs in Table 1 are listed in the last six columns of Table 2. We denote these vectors by $F A_{3}^{c}$ to stress that they correspond to child designs. The six different $A_{3}$ values obtained for the 563 -factor projections of the three designs were $2,2 / 3,14 / 27,4 / 9,8 / 27$ and 0 . The value of 2 is particularly unfavorable. It means that, in the corresponding three-factor projection, the main effect of any one factor is completely aliased with a component of the two-factor interaction of the two other factors. 
The child designs obtained from parent designs 1 and 2 both have a frequency distribution equal to $F A_{3}^{c}=(2,18,0,0,0,36)$. For the child design from parent design 3 , the frequency distribution is given by $F A_{3}^{c}=(0,1,11,16,23,5)$. So, the worst three-factor projections of the child designs from parent designs 1 and 2 have $A_{3}$ values of 2 and $2 / 3$. Two of the 56 projections into three factors have an $A_{3}$ value of 2 , and 18 of the projections have an $A_{3}$ value of $2 / 3$. The child design from parent design 3 has better projection properties because none of its projections into three factors has an $A_{3}$ value of 2 , and only one of its projections has an $A_{3}$ value of $2 / 3$. In general, designs that have small frequencies for large $A_{3}$ values are preferable. The design that has the best projection properties, as measured by the $F A_{3}$ distribution, is said to have minimum projection aberration or minimum PA. Clearly, the child design from parent design 3 is the better option in Table 2 in terms of the PA criterion.

What is most interesting about the GA and PA criteria is that they favor different designs. The child designs from parents 1 and 2 are the best in terms of the GA criterion, whereas the child design from parent 3 is better in terms of the PA criterion.

In Table 2, the last element of the $F A_{3}^{c}$ vector gives the frequency of three-factor projections with an $A_{3}$ value of zero. In the remainder of this paper, we omit this frequency, because it is redundant. Indeed, it can always be calculated as the difference between $k(k-1)(k-2) / 6$ and the sum of all other frequencies in the $F A_{3}$ vector.

\section{Blocking optimality}

A key feature of screening experiments is that not all the main effects and two-factor interaction effects can be estimated simultaneously. When the experiment is blocked, this undesirable feature of screening experiments is even worse because a proper data analysis requires the estimation of block effects on top of the main effects of the treatment factors and the two-factor interaction effects. In this paper, we consider only blocking arrangements for which the main effects of the treatment factors can be estimated independently from the block effects. This is because we wish to prioritize estimation of main effects. In a blocked experiment, besides being aliased with main-effect contrast vectors and other two-factor interaction contrast vectors, any two-factor interaction contrast vector can also be confounded with block contrast vectors. As a result, the challenge is to find blocking patterns that have as little aliasing as possible between main-effect contrast vectors and two-factor interaction contrast vectors and among the two-factor interaction contrast vectors (as in the absence of blocking), and an optimal confounding pattern between the two-factor interaction contrast vectors and the block contrast vectors. 


\subsection{Pure- and mixed-type words}

When a design involves a blocking factor, there are contrast vectors involving the blocking factor and those that do not involve the blocking factor. Therefore, any $A_{i}$ value of a parent design, which we denote by $A_{i}^{p}$ (where the superscript ' $p$ ' is short for parent design), partly corresponds to the first type of contrast vectors and partly to the second type. Following Cheng and Wu (2002), we call the portion of $A_{i}$ that corresponds to contrasts involving the blocking factor mixed-type word counts and the portion of $A_{i}$ that corresponds to contrasts not involving the blocking factor pure-type word counts. This distinction is important for the evaluation of a given blocking arrangement, because it is less important to have low mixed-type word counts than it is to have low pure-type word counts. This is due to the fact that blocking factors are assumed not to interact with treatment factors. Mixed-type word counts quantify the extent to which there is confounding between the treatment factors' interaction contrast vectors on the one hand, and block contrast vectors on the other hand, but also to what extent there is confounding between treatment $\times$ block contrast vectors and treatment contrast vectors. Mixed-type word counts are therefore only partially relevant.

A crucial insight for the purpose of this paper is that the pure-type word count of length $i$ of a parent design equals $A_{i}^{c}$, the word count of length $i$ of the child design. In the remainder of this paper, we denote the mixed-type word count of length $i$ by $A_{i-1,1}$. It is easy to see that $A_{i}^{p}=A_{i}^{c}+A_{i-1,1}$.

\section{$3.2 \quad$ Five criteria}

There has been extensive discussion in the literature about the importance of mixed-type and pure-type word counts in design selection criteria. An overview of this discussion is given in Cheng et al. (2004) and Ai and Zhang (2004). While all authors agree that the $A_{3}^{c}$ value should be minimized first, there is considerable debate about the order of importance of the $A_{4}^{c}, A_{21}$, and $A_{31}$ values.

Assuming that words of length $i \geq 5$ can be ignored, we identified five sensible design selection criteria. The first four criteria are based on two different orders of importance of the $A_{3}^{c}, A_{4}^{c}, A_{21}$, and $A_{31}$ values, while the fifth criterion is based on the properties of the designs when projected into three factors. We list the five criteria in Table 3. Using the criteria requires minimizing the entries in the table from left to right.

\section{$W_{1}$ - and $W_{2}$-criteria}

The criteria labeled $W_{1}$ and $W_{2}$ in Table 3 were used by Cheng and Wu (2002) and Cheng et al. (2004). These criteria are classical criteria because they both seek to minimize the most severe types of aliasing and confounding. Which of the two criteria is preferable depends on the experimental context. Sometimes, confounding of two-factor interaction effects with blocks is considered more serious than aliasing among 
Table 3: Five criteria for selecting optimal blocking arrangements. Entries have to be minimized from left to right.

\begin{tabular}{lrrrr}
\hline Criterion & \multicolumn{4}{c}{ Minimization Vector } \\
\hline$W_{1}$ & $A_{3}^{c}$ & $A_{4}^{c}$ & $A_{21}$ & $A_{31}$ \\
$W_{2}$ & $A_{3}^{c}$ & $A_{21}$ & $A_{4}^{c}$ & $A_{31}$ \\
$W_{1}^{-}$ & $A_{3}^{c}$ & $A_{4}^{c}$ & $-A_{21}$ & $A_{31}$ \\
$W_{2}^{-}$ & $A_{3}^{c}$ & $-A_{21}$ & $A_{4}^{c}$ & $A_{31}$ \\
$W_{3}$ & $F A_{3}^{c}$ & $F A_{21}$ & & \\
\hline
\end{tabular}

two-factor interaction effects. In that case, criterion $W_{2}$ is preferred. In other experiments, criterion $W_{1}$ might be the preferred criterion.

\section{$W_{1}^{-}$- and $W_{2}^{-}$-criteria}

To the best of our knowledge, the $W_{1}^{-}$- and $W_{2}^{-}$-criteria are new to the literature. The novel feature of these criteria is that they seek to maximize the confounding between the two-factor interaction effects and the blocking factor, while they still aim at minimizing the aliasing of main effects of treatment factors with their two-factor interactions, aliasing of two-factor interactions with other two-factor interactions and confounding of three-factor interactions with blocks. In Table 3 , we have made this clear by using the entry $-A_{21}$ instead of $A_{21}$ for the $W_{1}^{-}$- and $W_{2}^{-}$-criteria. As a matter of fact, minimizing $-A_{21}$ is the same as maximizing the mixed-type word count of length $3, A_{21}$.

The $W_{1}^{-}$- and $W_{2}^{-}$-criteria are useful when we use a main-effects only model to detect active factors, in cases with insufficient degrees of freedom to estimate two-factor interaction effects. This is because, when the $A_{21}$ value is large, there are fewer two-factor interaction effects that may inflate the residual error variance in a model involving block effects and main effects. In that case, all other things being equal, the power for detecting active factors is increased.

We illustrate the advantage of maximizing $A_{21}$ with a small simulation study that involves the first two designs of Table 1. Both designs are arrangements of an eight-factor 27-run design in three blocks of size nine. The design with ID 1 in the table is $W_{1}$-optimal, whereas the design with ID 2 is $W_{1}^{-}$-optimal. For each of the two designs, we first normalized the interaction contrast vectors to a length of one. We then generated 5000 sets of responses assuming that the two-factor interaction coefficients are from a standard normal distribution. For each of the 5000 data sets, we estimated a model including block effects and main effects, and quantified the mean squares value for the blocks, the main effects and the residual error. Side-by-side box plots of the mean squares for the 5000 data sets are shown in Figure 1.

The figure shows that using the $W_{1}^{-}$-optimal blocking arrangement leads to a larger value of the mean 


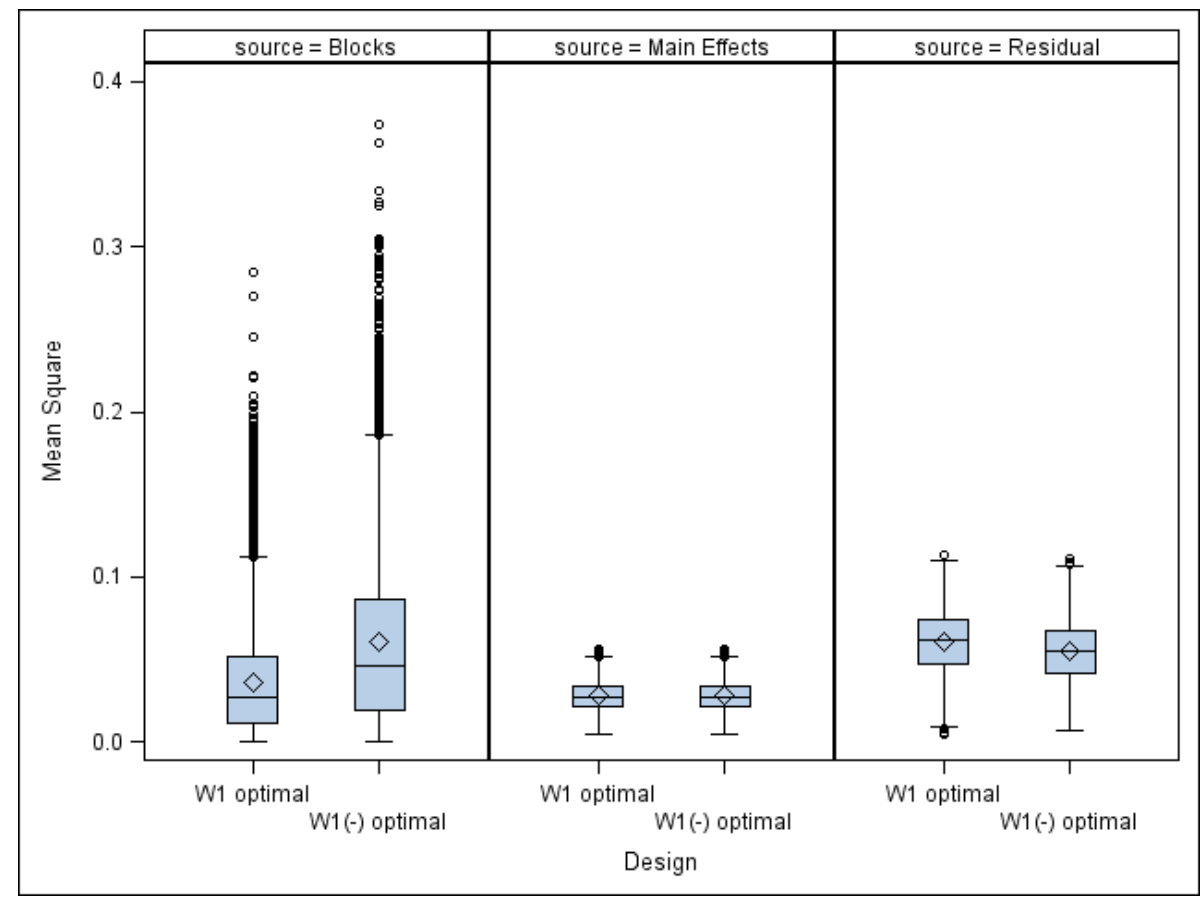

Figure 1: Comparison of mean squares for blocks, main effects and residual error for a $W_{1}$-optimal and a $W_{1}^{-}$-optimal blocking arrangement of a 27-run design involving eight three-level factors, assuming a model involving block effects and main effects is fitted.

squares for the blocks than the $W_{1}$-optimal blocking arrangement. Conversely, the $W_{1}^{-}$-optimal blocking arrangement leads to a smaller value of the mean squares for the residual error than the $W_{1}$-optimal blocking arrangement. Finally, the mean squares for the main effects remain unchanged. In $56 \%$ of the simulations, the error mean squares for the $W_{1}$-optimal design were higher than the error mean squares for the $W_{1}^{-}$optimal alternative. In $50 \%$ of the simulations, the increase in mean squares was $11 \%$ or more. A drop in the mean squares for the residual error results in a smaller estimate for the residual error variance, and therefore in a larger value for the test statistics for the main effects. As a result, the power for detecting main effects increases. For this reason, the $W_{1}^{-}$-optimal blocking arrangement is more suitable for detecting active main effects than the $W_{1}$-optimal arrangement. Since the child designs of the $W_{1}$ - and $W_{1}^{-}$-optimal designs are isomorphic in this example, the differences in mean squares are entirely due to the blocking.

Note that, in Figure 1, the drop in the mean squares for the residual error is smaller than the increase in the mean squares for the blocks. This is due to the fact that, in the main-effects model we utilized to analyze the simulated data, eight degrees of freedom are associated with the mean squares for the residual error, while only two degrees of freedom are associated with blocks. In a case with a larger number of blocks and a smaller number of degrees of freedom for the residual error, the decrease in the mean squares value for the residual error would be larger relative to the increase in the mean squares value for the blocks. 
Table 4: GWLP-based characteristics of the blocked designs in Table 1.

\begin{tabular}{lllllll}
\hline ID & $A_{3}^{c}$ & $A_{4}^{c}$ & $A_{21}$ & $A_{31}$ & $A_{3}^{p}$ & $A_{4}^{p}$ \\
\hline 1 & 16 & 60 & 8 & 48 & 24 & 108 \\
2 & 16 & 60 & 14 & 24 & 30 & 84 \\
3 & 20.30 & 45.85 & 10.11 & 36.82 & 30.41 & 82.67 \\
\hline
\end{tabular}

\section{$W_{3}$-criterion}

The final criterion we use is based on the projections of designs into three factors. We label that criterion $W_{3}$. The $W_{3}$-criterion is similar in spirit to the PA criterion discussed in the Section 2, but it distinguishes between the frequency vector for the pure-type word counts of length $3, F A_{3}^{c}$, and the frequency vector for the mixed-type word counts of that length, $F A_{21}$. More specifically, the $W_{3}$-criterion requires sequentially minimizing the elements of $F A_{3}^{c}$, followed by sequentially minimizing the elements of $F A_{21}$. The criterion is a simplified version of the $W_{2}^{G}$-criterion proposed by Cheng et al. (2004) for blocking two-level designs.

\section{Application}

To illustrate the application of the five blocking criteria, consider again the three designs in Table 1. The GWLP-based characteristics of these designs are shown in Table 4. Of the three designs, the one labeled 1 performs best in terms of $W_{1^{-}}$and $W_{2}$-criteria. In terms of the $W_{1}^{-}$- and $W_{2}^{-}$-criteria, the design labeled 2 is best.

The word-length frequency vectors required to determine the designs that are best with respect to the $W_{3}$-criterion are given in Table 5 . Of the three designs, the design labeled 3 is best according to the puretype word-length frequency vector $F A_{3}^{c}$, because it has a zero frequency for $A_{3}=2$. As a result, for each projection into three factors, the model with all main effects and all two-factor interaction effects is estimable. Design 2 has a zero frequency of $A_{3}=2$ in the mixed-type word-length frequency vector $F A_{21}$. Although that design has a frequency of two for $A_{3}=2$ in the pure-type word-length frequency vector $F A_{3}^{c}$, it is a compromise between the two other designs, because it has a better $F A_{21}$ vector than design 1 (see Table 5) and a better $A_{3}^{c}$ value than design 3 (see Table 4).

\section{Finding optimal blocking arrangements}

In this section, we explain how to determine the optimal blocking arrangements according to the $W_{1^{-}}, W_{1}^{-}$,

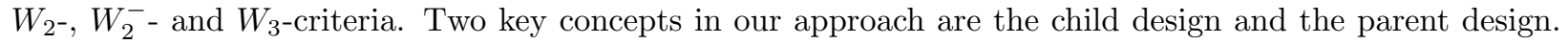
The child design is an orthogonal array denoted by $\mathrm{OA}\left(N ; s_{1} \ldots s_{k}\right)$. The parent design is an orthogonal 
Table 5: Pure-type and mixed-type word-length frequency vectors $F A_{3}^{c}, F A_{21}$ and $F A_{3}^{p}$ for the three-factor projections of the designs in Table 1.

\begin{tabular}{lrrrrrr}
\hline & & \multicolumn{5}{c}{$A_{3}$ values } \\
\cline { 3 - 7 } ID & & 2 & $2 / 3$ & $14 / 27$ & $4 / 9$ & $8 / 27$ \\
\hline \multirow{1}{*}{1} & $F A_{3}^{c}$ & 2 & 18 & 0 & 0 & 0 \\
& $F A_{21}$ & 1 & 9 & 0 & 0 & 0 \\
\cline { 2 - 7 } 2 & $F A_{3}^{p}$ & 3 & 27 & 0 & 0 & 0 \\
& $F A_{3}^{c}$ & 2 & 18 & 0 & 0 & 0 \\
& $F A_{21}$ & 0 & 7 & 14 & 0 & 7 \\
\hline \multirow{3}{*}{3} & $F A_{3}^{p}$ & 2 & 25 & 14 & 0 & 7 \\
& $F A_{3}^{c}$ & 0 & 1 & 11 & 16 & 23 \\
& $F A_{21}$ & 0 & 1 & 7 & 5 & 13 \\
\cline { 2 - 7 } & $F A_{3}^{p}$ & 0 & 2 & 18 & 21 & 36 \\
\hline
\end{tabular}

array with one extra column for the blocking factor. Since we denote the number of blocks by $b$, that extra column should have $b$ levels. We denote this kind of array by $\mathrm{OA}\left(N ; s_{1} \ldots s_{k} b\right)$. This is important because all possible ways of blocking $k$-factor child designs can be obtained by enumerating all non-isomorphic parent designs of the type $\mathrm{OA}\left(N ; s_{1} \ldots s_{k} b\right)$, and studying all possible projections of these parent designs into designs with one fewer $b$-level column. A key feature of this approach, which is known as single replacement in the literature, is that it ensures orthogonality of the blocking factor with respect to the main effects of the $k$ treatment factors. This is consistent with our prioritization of the main-effects estimation.

Our use of single replacement for any number of blocks $b$ is possible due to the work of Schoen et al. (2010), whose enumeration algorithm enabled us to obtain complete catalogs of all non-isomorphic pure-level and mixed-level orthogonal arrays, which can serve as parent and child designs.

\subsection{Determining $A_{3}^{c}, A_{21}, A_{4}^{c}$ and $A_{31}$ values and $F A_{3}^{c}$ and $F A_{21}$ vectors}

The first step in finding optimal blocking arrangements is to determine the $A_{3}^{c}, A_{21}, A_{4}^{c}$ and $A_{31}$ values and the pure-type and mixed-type word-length frequency vectors $F A_{3}^{c}$ and $F A_{21}$. We start by computing the $A_{3}^{p}$ and $A_{4}^{p}$ values, and the word length frequency vector, $F A_{3}^{p}$, of the parent designs. The challenge then is to split the $A_{3}^{p}$ values into $A_{3}^{c}$ and $A_{21}$ values, the $A_{4}^{p}$ values into $A_{4}^{c}$ and $A_{31}$ values, and the $F A_{3}^{p}$ vectors into $F A_{3}^{c}$ and $F A_{21}$ vectors. The most convenient way to achieve this is by computing the $A_{3}$ and $A_{4}$ values of any possible child design that can be obtained from the parent designs by dropping a $b$-level factor. The $A_{21}$ and $A_{31}$ values are then calculated as $A_{21}=A_{3}^{p}-A_{3}^{c}$ and $A_{31}=A_{4}^{p}-A_{4}^{c}$, respectively. In a similar fashion, 
we computed the mixed-type word-length frequency vector $F A_{21}$ as the element-wise difference between the $F A_{3}$ vectors of the parent designs and those of the child designs.

We illustrate this approach for parent design 1 in Table 1. This design involves nine three-level factors in total, eight treatment factors and one blocking factor. The nine-factor design is a parent design with $A_{3}^{p}=24$ and $A_{4}^{p}=108$. Its $F A_{3}^{p}$ frequency vector is $(3,27,0,0,0)$, with elements corresponding to the $A_{3}$ values $2,2 / 3,14 / 27,4 / 9$ and $8 / 27$ for the three-factor projections. As shown in Table 2 , the child design obtained by dropping the blocking factor for design 1 in Table 1 has $A_{3}^{c}=16$ and $A_{4}^{c}=60$. Therefore, the blocking arrangement for design 1 in Table 1 has $A_{21}=24-16=8$ and $A_{31}=108-60=48$.

Table 2 also shows that the $F A_{3}^{c}$ frequency vector is $(2,18,0,0,0)$. As a result, the frequency vector for the mixed-type counts, $F A_{21}$, is $(3-2,27-18,0,0,0)$ or $(1,9,0,0,0)$. This is shown in Table 5, along with the results for parent designs 2 and 3 .

\subsection{Determining the optimal blocking arrangements}

Design 1 in Table 1 is a minimum GA design for nine three-level factors and 27 runs. In addition, its eight-factor projection obtained by dropping the blocking factor is a minimum GA design for eight threelevel factors and 27 runs. Therefore, the design sequentially minimizes $\left(A_{3}^{c}, A_{4}^{c}, A_{3}^{p}, A_{4}^{p}\right)$. This is equivalent to sequentially minimizing $\left(A_{3}^{c}, A_{4}^{c}, A_{3}^{p}-A_{3}^{c}, A_{4}^{p}-A_{4}^{c}\right)$, or $\left(A_{3}^{c}, A_{4}^{c}, A_{21}, A_{31}\right)$. As a result, the blocking arrangement given in Table 1 for design 1 is the best blocking arrangement in terms of the $W_{1}$-criterion.

The blocking arrangement obtained from parent design 1 is also optimal in terms of the $W_{2}$-criterion. Optimization of this criterion requires sequential minimization of $\left(A_{3}^{c}, A_{21}, A_{4}^{c}, A_{31}\right)$, which is equivalent to sequential minimization of $\left(A_{3}^{c}, A_{3}^{p}, A_{4}^{c}, A_{4}^{p}\right)$. The fact that the blocking arrangement is optimal in terms of both the $W_{1}$-criterion and the $W_{2}$-criterion is due to the fact that all eight-factor designs with minimum $A_{3}^{c}$ value have the same $A_{4}^{c}$ value. Therefore, sequentially minimizing $\left(A_{3}^{c}, A_{4}^{c}, A_{3}^{p}, A_{4}^{p}\right)$ to find the best design in terms of the $W_{1}$-criterion gives exactly the same results as sequentially minimizing $\left(A_{3}^{c}, A_{3}^{p}, A_{4}^{c}, A_{4}^{p}\right)$ to find the best design in terms of the $W_{2}$-criterion. However, it will not generally be the case that the $W_{1^{-}}$and $W_{2}$-criteria favor the same design.

The nine-factor parent design 2 in Table 1 has $A_{3}^{p}$ and $A_{4}^{p}$ values of 30 and 84, respectively. Dropping the blocking factor of the design results in a minimum GA design for eight factors. Design 2 has the worst possible $A_{3}^{p}$ value among all parent designs that project into the minimum GA eight-factor child design. So, the blocking arrangement is such that the vector $\left(A_{3}^{c}, A_{4}^{c},-A_{3}^{p}, A_{4}^{p}\right)$ is sequentially minimized. Therefore, it is optimal in terms of the $W_{1}^{-}$-criterion. Due to the fact that all eight-factor designs with minimum $A_{3}^{c}$ value have the same $A_{4}^{c}$ value, the blocking arrangement is also optimal in terms of the $W_{2}^{-}$-criterion.

Finally, the nine-factor parent design 3 in Table 1 has an $F A_{3}^{p}$ vector equal to $(0,2,18,21,36)$. It therefore 
sequentially minimizes the $F A_{3}^{p}$ vector. Dropping the blocking factor of the design results in an eight-factor projection that is ranked third in the $F A_{3}$ ranking of all eight-factor child designs. The resulting eight-factor child design is, however, the best ranked one among all eight-factor designs that can be obtained from any nine-factor design by means of projection. In other words, it sequentially minimizes the $F A_{3}^{c}$ vector among all child designs. Because parent design 3 has the best $F A_{3}^{p}$ ranking among all possible nine-factor parent designs and projects into the best child design, its blocking arrangement in Table 1 sequentially minimizes $\left(F A_{3}^{c}, F A_{3}^{p}\right)$. This is equivalent to minimizing $\left(F A_{3}^{c}, F A_{3}^{p}-F A_{3}^{c}\right)$ or $\left(F A_{3}^{c}, F A_{21}\right)$. Using the blocking factor of design 3 therefore leads to the best blocking arrangement according to the $W_{3}$-criterion. The $F A_{21}$ frequency vector is $(0,2-1,18-11,21-16,36-23)$ or $(0,1,7,5,13)$.

The different minimization vectors in Table 3 make it easy to determine optimal blocking arrangements. For given numbers of treatment factors $k$ and numbers of levels $s_{1}, \ldots, s_{k}$, we first create all non-isomorphic parent designs of the type $\mathrm{OA}\left(N ; s_{1} \ldots s_{k} b\right)$ and determine their $A_{3}^{p}, A_{4}^{p}$ and $F A_{3}^{p}$ values. We then create all possible child designs by dropping a $b$-level factor and determine their $A_{3}^{c}$ and $A_{4}^{c}$ values, the corresponding $A_{21}$ and $A_{31}$ values, the $F A_{3}^{c}$ vector and the corresponding $F A_{21}$-vector. The optimal blocking arrangements according to the $W_{1^{-}}, W_{1}^{-}-, W_{2^{-}}, W_{2}^{-}$- and $W_{3^{-}}$criteria can then be found by sequentially minimizing the entries in Table 3.

In many of the scenarios we investigated, we found that there are several blocking arrangements which have the same values for $A_{3}^{c}, A_{4}^{c}, A_{3}^{p}$ and $A_{4}^{p}$, but different word-length frequency vectors $F A_{3}^{c}$ and $F A_{3}^{p}$. Generally, designs that sequentially minimize the $F A_{3}^{c}$ and $F A_{3}^{p}$ vectors increase the quality of the threefactor projections. Therefore, we used these vectors as tie-breakers to distinguish between designs that are optimal in terms of the $W_{1^{-}}, W_{1}^{-}-, W_{2^{-}}$and $W_{2}^{-}$-criteria. Of all $W_{1^{-}}, W_{1}^{-}-, W_{2^{-}}$and $W_{2}^{-}$-optimal designs, we only report the ones that minimize the $F A_{3}^{c}$ and $F A_{3}^{p}$ vectors. This explains why, in a few cases, we report fewer designs than Cheng et al. (2004).

\section{Optimal blocking arrangements for designs with 12-27 runs}

In this section, we apply our blocking method to catalogs of all non-isomorphic orthogonal designs with $12,16,18,20$, and 27 runs that include at least one factor at more than two levels. We do not address arrangements of two-level designs in two blocks because these were studied earlier by Cheng et al. (2004, CLY). However, we do address the arrangement of two-level 16-run designs in four blocks, because these can be derived from designs of the type $\mathrm{OA}\left(16 ; 4^{1} 2^{n}\right)$. We use this type of design to point out differences between our methodology and CLY's methodology. Files containing all optimal blocking arrangements reported in this section are available electronically from the authors. 
The design tables in this section identify all parent designs by three basic characteristics. First, a simple ID is given. Second, the $R_{1}$ ranking gives the rank of a design after sequentially sorting the designs in ascending order of the $A_{3}$ and $A_{4}$ values and the $F A_{3}$ vector. Third, the $R_{2}$ ranking gives the rank of a design after sequential sorting the designs in ascending order of the $F A_{3}$ vector and the $A_{4}$ value. The designs with $R_{1}=1$ are recommended when more than three active factors are expected. The designs with $R_{2}=1$ are recommended when at most three factors are expected to be active.

In the tables, the basic characteristics of the designs are followed by the $A_{3}$ and $A_{4}$ values and the $F A_{3}$ vector. Finally, each table involves one or two columns describing the blocking arrangements that can be obtained from the parent designs. In Section 5.1, we explain in detail how the tables with optimal blocking arrangements can be used.

\subsection{Designs with 12 runs}

Wang and $\mathrm{Wu}(1992)$ were the first to list the complete series of non-isomorphic designs of the type OA(12; $3 \times$ $2^{n}$ ). Table 6 shows the designs we recommend for blocking purposes. The $R_{1}$ and $R_{2}$ rankings of the designs are identical. The $A_{3}$ values for three-factor projections corresponding to the three elements of the $F A_{3}$ vector are $1,2 / 3$, and $1 / 9$.

A design of the type $\mathrm{OA}\left(12 ; 3 \times 2^{n}\right)$ can be used to arrange a $3 \times 2^{n-1}$ design in two blocks of size 6 , or to arrange a $2^{n}$ design in three blocks of size 4 . The columns labeled ' 2 blocks' and ' 3 blocks' give the blocking arrangements that are optimal with respect to each of the five optimality criteria we defined. We

Table 6: Optimal blocking arrangements based on parent designs of the type $\mathrm{OA}\left(12 ; 3 \times 2^{n}\right)$. Elements of the $F A_{3}$ vector correspond to $A_{3}$ values of $1,2 / 3$, and $1 / 9$. An ' $\mathrm{x}$ ' indicates $W_{1}$ - and $W_{2}$-optimal arrangements; a ' $y$ ' indicates $W_{1}^{-}$- and $W_{2}^{-}$-optimal arrangements; a 'z' indicates $W_{3}$-optimal arrangements.

\begin{tabular}{|c|c|c|c|c|c|c|c|c|c|}
\hline$n$ & ID & $R_{1}, R_{2}$ & $A_{3}$ & $A_{4}$ & \multicolumn{3}{|c|}{$F A_{3}$} & 2 blocks & 3 blocks \\
\hline 4 & 1 & 1 & 1.778 & 1 & 0 & 2 & 4 & 1:2;xyz & xyz \\
\hline 3 & 1 & 1 & 0.111 & 0.889 & 0 & 0 & 1 & $1: 1 ; \mathrm{xz}$ & $\mathrm{xz}$ \\
\hline & 2 & 2 & 0.778 & 0.222 & 0 & 1 & 1 & & \\
\hline 2 & 1 & 1 & 0 & - & 0 & 0 & 0 & $1: 1 ; x z$ & $\mathrm{xz}$ \\
\hline
\end{tabular}

Table 7: Properties of two-level child designs obtained from parent designs of the type $\mathrm{OA}\left(12 ; 3 \times 2^{n}\right)$. Elements of the $F A_{3}$ vector correspond to $A_{3}$ values of $1,2 / 3$, and $1 / 9$.

\begin{tabular}{rrrrccc}
\hline$n$ & $R_{1}^{p}$ & $A_{3}$ & $A_{4}$ & \multicolumn{3}{c}{$F A_{3}$} \\
\hline 4 & 1 & 0.444 & 0.111 & 0 & 0 & 4 \\
3 & 1 & 0.111 & - & 0 & 0 & 1
\end{tabular}


explain how to use the table by example.

The entries in the column labeled ' 2 blocks' are of the form 'p:q; \{criteria\}'. The first digit, p, indicates which column of the parent design has to be used for the blocking factor. The second digit, q, is the $R_{1}$ rank of the child design obtained by dropping the column corresponding to the blocking factor, and \{criteria\} specifies the set of criteria for which the resulting blocking arrangement is optimal. Consider, first, the single design of the type $\mathrm{OA}\left(12 ; 3 \times 2^{4}\right)$. It is impossible to project this design into the design of the type $\mathrm{OA}\left(12 ; 3 \times 2^{3}\right)$ with $R_{1}=1$ by dropping one of its two-level columns. However, by dropping the first two-level column, the $\mathrm{OA}\left(12 ; 3 \times 2^{4}\right)$ design projects into the $\mathrm{OA}\left(12 ; 3 \times 2^{3}\right)$ design with $R_{1}=2$. This explains the entry ' $1: 2$ ' in the column labeled ' 2 blocks'. The $\mathrm{OA}\left(12 ; 3 \times 2^{3}\right)$ child design is reported in Table 6 as well, in the row for $n=3$ and $R_{1}=2$. Dropping any other two-level column than the first one from the $\mathrm{OA}\left(12 ; 3 \times 2^{4}\right)$ design also results in the $\mathrm{OA}\left(12 ; 3 \times 2^{3}\right)$ child design with $R_{1}=2$. So, there is a single parent design, and it projects into a single child design. Therefore, the blocking arrangement is optimal according to all five optimality criteria considered here. This is indicated by the letters $\mathrm{x}, \mathrm{y}$, and $\mathrm{z}$ after the semicolon in the column labeled ' 2 blocks'. The $A_{21}$ value and the $F A_{21}$ vector for this blocking arrangement can be found by subtracting the $A_{3}$ value and the $F A_{3}$ frequencies of the child design from the $A_{3}$ value and the $F A_{3}$ frequencies of the parent design, respectively. This yields $A_{21}=1.778-0.778=1$ and $F A_{21}=(0-0,2-1,4-1)=(0,1,3)$.

By dropping the three-level factor of the $\mathrm{OA}\left(12 ; 3 \times 2^{4}\right)$ parent design, we obtain a child design of the type $\mathrm{OA}\left(12 ; 2^{4}\right)$. We have displayed the $A_{3}$ and $A_{4}$ values and the $F A_{3}$ vectors of the child design in Table 7 . The $2^{4}$ child design has $A_{3}$ and $A_{4}$ values of 0.444 and 0.111 , respectively, and an $F A_{3}$ vector equal to $(0,0,4)$. Again, the $A_{21}$ value and the $F A_{21}$ vector can be found by subtracting the entries of the child design from those of the parent design. This yields $A_{21}=1.778-0.444=1.333$ and $F A_{21}=(0-0,2-0,4-4)=(0,2,0)$.

There are three different designs of the type $\mathrm{OA}\left(12 ; 3 \times 2^{3}\right)$. Two of these are listed in Table 6 . Their two-level parts consist of a full $2^{3}$ design combined with one regular half-fraction of that design. The $\mathrm{OA}\left(12 ; 3 \times 2^{3}\right)$ design with $R_{1}=1$ can be used to obtain the best blocking arrangement in terms of the $W_{1^{-}}$,

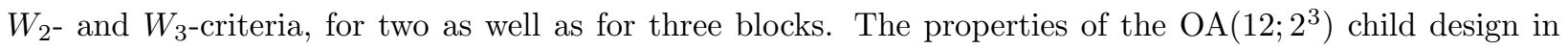
the three-blocks case are given in Table 7 . The $\mathrm{OA}\left(12 ; 3 \times 2^{3}\right)$ design with $R_{1}=2$ is reported only because it is the child design corresponding to the $\mathrm{OA}\left(12 ; 3 \times 2^{4}\right)$ parent design. In other words, the $\mathrm{OA}\left(12 ; 3 \times 2^{3}\right)$ design with $R_{1}=2$ can be arranged in two blocks of size 6 , using the $\mathrm{OA}\left(12 ; 3 \times 2^{4}\right)$ as a parent design.

Using the three-level factor of the $\mathrm{OA}\left(12 ; 3 \times 2^{3}\right)$ design with $R_{1}=2$ as a blocking factor results in an optimal blocking arrangement in terms of the $W_{1}^{-}$- and $W_{2}^{-}$-criteria. However, we do not recommend these criteria in this case, for the following reason. The rationale of the $W_{1}^{-}-$and $W_{2}^{-}$-criteria is to minimize the inflation of the residual error by two-factor interactions in a model containing main effects and block effects 
only. However, any $\mathrm{OA}\left(12 ; 2^{3}\right)$ design arranged in three blocks of size four allows us to fit a model containing all main effects and all two-factor interactions, with three degrees of freedom for estimating the residual error variance. Hence, in this case, the $W_{1}^{-}$- and $W_{2}^{-}$-criteria, which assume that there are not enough degrees of freedom for estimating a model including all two-factor interaction effects, are not needed.

The $\mathrm{OA}\left(12 ; 3 \times 2^{3}\right)$ design with $R_{1}=3$ is not shown in Table 6 , even though it can be used to obtain a $W_{1}^{-}$- and $W_{2}^{-}$-optimal arrangement of a $\mathrm{OA}\left(12 ; 3 \times 2^{2}\right)$ design in two blocks. Here too, we do not recommend the $W_{1}^{-}$- and $W_{2}^{-}$-criteria, because the number of runs is large enough to estimate the block effects, the main effects and the two-factor interaction effects, with one degree of freedom for the residual error variance.

Finally, there are two designs of the type $\mathrm{OA}\left(12 ; 3 \times 2^{2}\right)$. The recommended one is the full factorial design, which can be arranged in three blocks of size four by assigning the three replicates of the $2^{2}$ factorial design to the three blocks, and in two blocks of size six by assigning the two replicates of the $3 \times 2$ factorial design to the two blocks. The resulting blocking arrangements are optimal in terms of the $W_{1^{-}}, W_{2^{-}}$and $W_{3}$-criteria.

The second design of the type $\mathrm{OA}\left(12 ; 3 \times 2^{2}\right)$ results in blocking arrangements that are optimal in terms of the $W_{1}^{-}$- and $W_{2}^{-}$-criteria. However, these criteria again are not appropriate because the number of runs is sufficiently large to estimate a model including all two-factor interaction effects. Therefore, the second $\mathrm{OA}\left(12 ; 3 \times 2^{2}\right)$ design is not shown in Table 6 .

Table 8: Optimal blocking arrangements based on parent designs of the type OA $\left(16 ; 4^{3} 2^{n}\right)$. Elements of the $F A_{3}$ vector correspond to $A_{3}$ values of $3,1,1 / 2$, and $1 / 4$. An 'x' indicates $W_{1}$ - and $W_{2}$-optimal arrangements; a ' $\mathrm{y}$ ' indicates $W_{1}^{-}$- and $W_{2}^{-}$-optimal arrangements; a ' $\mathrm{z}$ ' indicates $W_{3}$-optimal arrangements.

\begin{tabular}{|c|c|c|c|c|c|c|c|c|c|c|c|}
\hline$n$ & ID & $R_{1}$ & $R_{2}$ & $A_{3}$ & $A_{4}$ & \multicolumn{4}{|c|}{$F A_{3}$} & 2 blocks & 4 blocks \\
\hline 6 & 1 & 1 & 1 & 32 & 51 & 1 & 21 & 12 & 8 & 1:1;xyz & $1: 1 ; x y z$ \\
\hline 5 & 1 & 1 & 1 & 25 & 32 & 1 & 17 & 8 & 4 & 4:1;xyz & $1: 1 ; x y z$ \\
\hline 4 & 1 & 1 & 2 & 19 & 18 & 1 & 14 & 4 & 0 & & $3: 1 ; x y$ \\
\hline & 2 & 3 & 1 & 19 & 18.5 & 1 & 13 & 5 & 2 & $1: 3 ; \mathrm{z}$ & $1: 2 ; \mathrm{z}$ \\
\hline & 3 & 4 & 3 & 19 & 19 & 1 & 16 & 0 & 0 & 1:1;xy & \\
\hline 3 & 1 & 1 & 2 & 13 & 12 & 1 & 10 & 0 & 0 & $1: 1 ; \mathrm{xz}$ & $1: 2 ; \mathrm{x}$ \\
\hline & 2 & 2 & 1 & 13.75 & 9.75 & 1 & 9 & 3 & 1 & & \\
\hline & 3 & 3 & 3 & 14 & 9 & 1 & 10 & 2 & 0 & & 1:1;yz \\
\hline & 4 & 4 & 4 & 14 & 9 & 1 & 11 & 0 & 0 & $1: 1 ; y$ & \\
\hline 2 & 1 & 1 & 1 & 9 & 5 & 1 & 6 & 0 & 0 & $1: 1 ; \mathrm{xz}$ & $1: 1 ; x z$ \\
\hline & 2 & 3 & 3 & 10 & 3 & 1 & 7 & 0 & 0 & $1: 1 ; y$ & $1: 1 ; y$ \\
\hline 1 & 1 & 1 & 1 & 6 & 1 & 1 & 3 & 0 & 0 & 1:1;xyz & $1: 1 ; x y z$ \\
\hline 0 & 1 & 1 & 1 & 3 & 0 & 1 & 0 & 0 & 0 & & $1: 1 ; x y z$ \\
\hline
\end{tabular}




\subsection{Designs with 16 runs}

The blocking of pure two-level 16-run designs has been studied extensively by CLY. In many studies, however, experimenters also want to include factors with more than two levels. Tables 8-10 present parent designs as well as optimal blocking arrangements for cases with two-level factors and one or more additional four-level factors. In addition, Table 10 also shows the optimal arrangements of pure two-level designs in four blocks. While these blocking arrangements have been studied by CLY already, we decided to report a table with the best blocking arrangements found using our five criteria for this case for three reasons. First, the table facilitates a comparison of our methodology with the methodology of CLY. Second, we found an attractive blocking arrangement for the well-known resolution- $52^{5-1}$ design that was not given by CLY. Third, two of our criteria were not used by CLY.

A design is listed in Tables $8-10$ if it is best according to the $R_{1}$ or $R_{2}$ ranking, if it yields an optimal blocking arrangement, or if it is the child design of an optimal blocking arrangement. Table 8 contains

Table 9: Optimal blocking arrangements based on parent designs of the type $\mathrm{OA}\left(16 ; 4^{2} 2^{n}\right)$. Elements of the $F A_{3}$ vector correspond to $A_{3}$ values of $1,1 / 2$, and $1 / 4$. An 'x' indicates $W_{1^{-}}$and $W_{2}$-optimal arrangements; a ' $y$ ' indicates $W_{1}^{-}$- and $W_{2}^{-}$-optimal arrangements; a ' $z$ ' indicates $W_{3}$-optimal arrangements.

\begin{tabular}{|c|c|c|c|c|c|c|c|c|c|c|}
\hline$n$ & ID & $R_{1}$ & $R_{2}$ & $A_{3}$ & $A_{4}$ & & $F A_{3}$ & & 2 blocks & 4 blocks \\
\hline 9 & 1 & 1 & 1 & 33 & 69 & 15 & 24 & 24 & $1 ; 1 ; x y z$ & 1:1;xyz \\
\hline 8 & 1 & 1 & 1 & 26 & 47 & 12 & 20 & 16 & $1: 1 ; x y, 3: 9 ; z$ & $2: 1 ; x y z$ \\
\hline \multirow[t]{3}{*}{7} & 1 & 1 & 3 & 20 & 30 & 10 & 16 & 8 & & $2: 1 ; x y, 1: 5 ; z$ \\
\hline & 2 & 9 & 1 & 20 & 31 & 10 & 14 & 12 & & \\
\hline & 3 & 10 & 4 & 20 & 31 & 11 & 12 & 12 & $3: 1 ; x y z$ & \\
\hline \multirow[t]{4}{*}{6} & 1 & 1 & 1 & 14 & 21 & 8 & 8 & 8 & $1: 1 ; \mathrm{xz}$ & \\
\hline & 2 & 5 & 8 & 15 & 17 & 9 & 12 & 0 & & $2: 1 ; x y$ \\
\hline & 3 & 12 & 14 & 15 & 18 & 12 & 4 & 4 & $1: 2 ; \mathrm{y}$ & \\
\hline & 4 & 16 & 6 & 15 & 18.5 & 9 & 9 & 6 & & $1: 3 ; z$ \\
\hline \multirow[t]{4}{*}{5} & 1 & 1 & 1 & 10 & 12 & 6 & 6 & 4 & $1: 2 ; \mathrm{z}$ & \\
\hline & 2 & 2 & 4 & 10 & 12 & 7 & 4 & 4 & $3: 1 ; x$ & \\
\hline & 3 & 12 & 10 & 11 & 9 & 7 & 8 & 0 & & $1: 1 ; x y z$ \\
\hline & 4 & 21 & 21 & 11 & 10 & 11 & 0 & 0 & $1: 1 ; y$ & \\
\hline \multirow[t]{4}{*}{4} & 1 & 1 & 8 & 6 & 8 & 6 & 0 & 0 & $1: 2 ; \mathrm{x}$ & $1: 2 ; \mathrm{x}$ \\
\hline & 2 & 2 & 1 & 6.5 & 7 & 4 & 4 & 2 & $1: 1 ; z$ & \\
\hline & 3 & 12 & 7 & 7.5 & 4.5 & 5 & 5 & 0 & & $1: 1 ; z$ \\
\hline & 4 & 19 & 19 & 8 & 4 & 8 & 0 & 0 & $1: 1 ; \mathrm{y}$ & $1: 1 ; y$ \\
\hline \multirow[t]{4}{*}{3} & 1 & 1 & 1 & 4 & 3 & 3 & 2 & 0 & $1: 1 ; \mathrm{xz}$ & $1: 1 ; \mathrm{xz}$ \\
\hline & 2 & 2 & 5 & 4 & 3 & 4 & 0 & 0 & $1: 1 ; \mathrm{x}$ & \\
\hline & 3 & 8 & 9 & 5 & 1 & 5 & 0 & 0 & $1: 1 ; y$ & \\
\hline & 4 & 10 & 10 & 6 & 1 & 6 & 0 & 0 & & $1: 1 ; y$ \\
\hline \multirow[t]{2}{*}{2} & 1 & 1 & 1 & 2 & 1 & 2 & 0 & 0 & $1: 1 ; \mathrm{xz}$ & $1: 1 ; \mathrm{Xz}$ \\
\hline & 2 & 3 & 3 & 3 & 0 & 3 & 0 & 0 & $1: 1 ; y$ & \\
\hline 1 & 1 & 1 & 1 & 1 & - & 1 & 0 & 0 & 1:1;xyz & $1: 1 ; \mathrm{XZ}$ \\
\hline
\end{tabular}


the results for designs of the type $\mathrm{OA}\left(16 ; 4^{3} 2^{n}\right)$. Such designs can be used to arrange designs of the type $\mathrm{OA}\left(16 ; 4^{2} 2^{n}\right)$ in four blocks of size four, or to arrange $\mathrm{OA}\left(16 ; 4^{3} 2^{n-1}\right)$ designs in two blocks of size eight. The $A_{3}^{c}$ and $A_{4}^{c}$ values, and the $F A_{3}^{c}$ vector, for the arrangements in two blocks can be found in Table 8 as well. The $A_{3}^{c}$ and $A_{4}^{c}$ values, and the $F A_{3}^{c}$ vector, for the arrangements in four blocks are given in Table 9 .

Even in the small series of $\mathrm{OA}\left(16 ; 4^{3} 2^{n}\right)$ designs, there is a remarkable differentiation in optimality features. For example, there are four non-isomorphic $\mathrm{OA}\left(16 ; 4^{3} 2^{3}\right)$ designs. The design with ranking $R_{2}=1$ does not yield an optimal blocking arrangement according to any of the criteria, while the remaining three designs are needed to obtain an optimal blocking arrangement for each of our five optimality criteria.

Table 9 presents the results for the designs of the type $\mathrm{OA}\left(16 ; 4^{2} 2^{n}\right)$. An $\mathrm{OA}\left(16 ; 4^{2} 2^{n}\right)$ design can be used to arrange $\mathrm{OA}\left(16 ; 4 \times 2^{n}\right)$ designs in four blocks and to arrange $\mathrm{OA}\left(16 ; 4^{2} 2^{n-1}\right)$ designs in two blocks. The child designs for the cases involving four blocks are given in Table 10, whereas those for the cases involving two blocks are given in Table 9 itself. For the $\mathrm{OA}\left(16 ; 4^{2} 2^{n}\right)$ parent design for eight two-level factors, i.e. for $n=8$, Table 9 gives two different optimal arrangements in two blocks, depending on the column used for the blocking factor. Using the design's first column for the blocking factor results in a blocking arrangement that is $W_{1^{-}}, W_{2^{-}}, W_{1}^{-}$and $W_{2}^{-}$-optimal, whereas using the design's third column for the blocking factor results in a blocking arrangement that is $W_{3}$-optimal. This explains the entry ' $1: 1 ; x y, 3: 9 ; z$ ' in Table 9 . Similarly, the first parent design for seven two-level factors $(n=7)$ gives two different optimal arrangements in four blocks.

Table 10 presents the results for designs of the type $\mathrm{OA}\left(16 ; 4 \times 2^{n}\right)$. The parent designs for optimal arrangements in four blocks have child designs whose characteristics are given in Table 11.

The arrangements in four blocks in Table 10 permit a direct comparison of our results with those of CLY. In Table 10, we mark the designs not found by these authors with an asterisk. Remarkably, our $W_{1}$-optimal design with five two-level factors is one of these designs. Its treatment factors form a regular resolution-5 $2^{5-1}$ design. The $W_{1}$-optimal blocking arrangement, however, is nonregular because its $F A_{21}$ vector is $(0,6,0)$, where the frequency of 6 corresponds to an $A_{3}$ value of 0.5 . The best blocking arrangement of the regular resolution-5 $2^{5-1}$ design reported by CLY has an $F A_{21}$ vector of $(1,4,0)$, and therefore confounds one twofactor interaction effect with the blocks. Our $W_{1}$-optimal design does not confound any two-factor interaction effect with the blocks completely, and is therefore preferable. We provide an extensive comparison of all other arrangements of 16-run two-level designs in four blocks we obtained with these of CLY in Appendix A.

The case involving five two-level factors $(n=5)$ and four blocks in Table 10 shows, for the first time in this paper, that $W_{1}^{-}$-optimal designs can differ from $W_{2}^{-}$-optimal designs. The $W_{1}$-optimal design for this case is also different from the $W_{2}$-optimal design.

We do not list $W_{1}^{-}$- or $W_{2}^{-}$-optimal designs for cases involving three and four two-level factors in four 
blocks, because it is possible to estimate all the main effects and two-factor interactions using the $W_{1^{-}}$or $W_{2}$-optimal designs. As a result, there is no added value in utilizing the $W_{1}^{-}$- or $W_{2}^{-}$-optimal blocking arrangements in these scenarios.

There is also a set of designs of the type $\mathrm{OA}\left(16 ; 8 \times 2^{n}\right)$ involving up to eight two-level factors, which can be used to run an $\mathrm{OA}\left(16 ; 8 \times 2^{n-1}\right)$ design in two blocks of size eight or an $\mathrm{OA}\left(16 ; 2^{n}\right)$ design in eight blocks of size two. The only non-isomorphic $\mathrm{OA}\left(16 ; 8 \times 2^{n}\right)$ designs with three, five, six, seven or eight two-level factors can be constructed by folding over an eight-run full factorial or fractional factorial design,

Table 10: Optimal blocking arrangements based on parent designs of the type $\mathrm{OA}\left(16 ; 4 \times 2^{n}\right)$. Elements of the $F A_{3}$ vector correspond to $A_{3}$ values of $1,1 / 2$, and $1 / 4$. An ' $\mathrm{x}$ ' indicates $W_{1}$ - and $W_{2}$-optimal arrangements; an ' $\mathrm{x}_{1}$ ' indicates $W_{1}$-optimal arrangements; an ' $\mathrm{x}_{2}$ ' indicates $W_{2}$-optimal arrangements; a 'y' indicates $W_{1}^{-}$and $W_{2}^{-}$-optimal arrangements; a ' $\mathrm{y}_{1}$ ' indicates $W_{1}^{-}$-optimal arrangements; a ' $\mathrm{y}_{2}$ ' indicates $W_{2}^{-}$-optimal arrangements; a ' $\mathrm{z}$ ' indicates $W_{3}$-optimal arrangements. Two-level designs in four blocks marked with an asterisk, and all mixed-level designs in two-blocks, were not found previously.

\begin{tabular}{|c|c|c|c|c|c|c|c|c|c|c|}
\hline$n$ & ID & $R_{1}$ & $R_{2}$ & $A_{3}$ & $A_{4}$ & & $F A_{3}$ & & 2 blocks & 4 blocks \\
\hline 12 & 1 & 1 & 1 & 34 & 87 & 6 & 24 & 64 & 1:1;xyz & xyz \\
\hline 11 & 1 & 1 & 1 & 27 & 62 & 3 & 24 & 48 & 1:1;xyz & xyz \\
\hline \multirow{2}{*}{10} & 1 & 1 & 1 & 21 & 42 & 1 & 24 & 32 & $1: 11 ; z$ & xyz \\
\hline & 2 & 16 & 2 & 21 & 43 & 3 & 18 & 36 & $5: 1 ; x y$ & \\
\hline \multirow[t]{3}{*}{9} & 1 & 1 & 3 & 15 & 30 & 3 & 12 & 24 & $1: 1 ; \mathrm{x}$ & \\
\hline & 2 & 11 & 1 & 16 & 26 & 0 & 24 & 16 & $1: 37 ; z$ & $\mathrm{xyz}$ \\
\hline & 3 & 23 & 2 & 16 & 28 & 1 & 18 & 24 & $4: 1 ; y$ & \\
\hline \multirow[t]{5}{*}{8} & 1 & 1 & 3 & 11 & 19 & 1 & 12 & 16 & & \\
\hline & 2 & 3 & 11 & 11 & 19 & 3 & 8 & 16 & $5: 1 ; \mathrm{x}$ & \\
\hline & 3 & 34 & 2 & 12 & 14 & 0 & 24 & 0 & & xyz \\
\hline & 4 & 37 & 1 & 12 & 16 & 0 & 18 & 12 & $2: 5 ; \mathrm{z}$ & \\
\hline & 5 & 66 & 28 & 12 & 18 & 4 & 8 & 16 & $1: 1 ; y$ & \\
\hline \multirow[t]{4}{*}{7} & 1 & 1 & 24 & 7 & 13 & 3 & 4 & 8 & $3: 1 ; x y$ & \\
\hline & 2 & 3 & 6 & 7.5 & 12 & 1 & 8 & 10 & $1: 3 ; \mathrm{z}$ & \\
\hline & 3 & 5 & 1 & 8 & 10 & 0 & 12 & 8 & & \\
\hline & 4 & 64 & 5 & 9 & 7 & 0 & 18 & 0 & & xyz \\
\hline \multirow[t]{5}{*}{6} & 1 & 1 & 46 & 3 & 11 & 3 & 0 & 0 & $1: 2 ; \mathrm{x}$ & $\mathrm{x}$ \\
\hline & 2 & 3 & 1 & 4.5 & 8 & 0 & 6 & 6 & & \\
\hline & 3 & 4 & 3 & 5 & 6 & 0 & 8 & 4 & $2: 1 ; \mathrm{yz}$ & \\
\hline & 4 & 46 & 7 & 6 & 3 & 0 & 12 & 0 & & $\mathrm{z}$ \\
\hline & 5 & 71 & 47 & 7 & 3 & 3 & 8 & 0 & & $\mathrm{y}^{*}$ \\
\hline \multirow[t]{5}{*}{5} & 1 & 1 & 1 & 2 & 5 & 0 & 4 & 0 & $1: 1 ; \mathrm{xz}$ & $\mathrm{x}_{2} \mathrm{Z}$ \\
\hline & 2 & 2 & 30 & 2 & 5 & 2 & 0 & 0 & & \\
\hline & 3 & 6 & 6 & 3 & 3 & 0 & 6 & 0 & & $\mathrm{x}_{1} \mathrm{y}_{1}^{*}$ \\
\hline & 4 & 43 & 48 & 4 & 2 & 4 & 0 & 0 & $1: 2 ; \mathrm{y}$ & \\
\hline & 5 & 50 & 38 & 6 & 1 & 6 & 0 & 0 & & $\mathrm{y}_{2}{ }^{*}$ \\
\hline \multirow[t]{3}{*}{4} & 1 & 1 & 1 & 1 & 2 & 0 & 2 & 0 & $1: 1 ; \mathrm{xz}$ & $\mathrm{xz}$ \\
\hline & 2 & 2 & 11 & 1 & 2 & 1 & 0 & 0 & & \\
\hline & 3 & 15 & 21 & 2 & 1 & 2 & 0 & 0 & $1: 1 ; y$ & \\
\hline 3 & 1 & 1 & 1 & 0 & 1 & 0 & 0 & 0 & $1: 1 ; \mathrm{xz}$ & $\mathrm{xz}$ \\
\hline
\end{tabular}


and linking each fold-over pair to a different level of the eight-level factor. There are two non-isomorphic $\mathrm{OA}\left(16 ; 8 \times 2^{4}\right)$ designs, both of which have an $A_{3}$ value of 6 . The two-level part of one of these designs is a replicated half fraction of the $2^{4}$ design. Therefore, this design has an $A_{4}$ value of 1 . The other design, which is the better of the two, has a full factorial $2^{4}$ design for the two-level factors, which results in a zero $A_{4}$ value. For that design, the eight-level factor can be constructed using any three two-factor interactions of the two-level factors.

In each case, there is only one possible way to obtain an orthogonally blocked design involving two blocks of size eight from the $\mathrm{OA}\left(16 ; 8 \times 2^{n}\right)$ design. That arrangement can be obtained by using any two-level factor as the blocking factor. In case $n=4$, the best blocking pattern is obtained from the $\mathrm{OA}\left(16 ; 8 \times 2^{4}\right)$ with a zero $A_{4}$ value. For constructing 16-run designs involving eight blocks of size two, there is only one possibility, that is by using the eight-level factor as the blocking factor. In case $n=4$, it is again best to use the $\mathrm{OA}\left(16 ; 8 \times 2^{4}\right)$ design with a zero $A_{4}$ value.

\subsection{Designs with 18 runs}

In this subsection, we provide optimal blocking arrangements of 18-run designs. These designs permit the study of up to seven three-level factors, and an additional two-level factor, and the study of one six-level factor with up to six three-level factors. The designs, denoted by $\mathrm{OA}\left(18 ; 3^{n} \times 2\right), \mathrm{OA}\left(18 ; 3^{n}\right)$ and $\mathrm{OA}\left(18 ; 6 \times 3^{n}\right)$, have been studied extensively before. Schoen (2009) provides a recent overview of the literature on 18-run designs and a comprehensive characterization of the designs. The blocking of these 18-run designs has,

Table 11: Properties of two-level child designs obtained from parent designs of the type $\mathrm{OA}\left(16 ; 4 \times 2^{n}\right)$. Elements of the $F A_{3}$ vector correspond to $A_{3}$ values of $1,1 / 2$, and $1 / 4$. The column labeled $R_{1}^{p}$ gives the $R_{1}$ ranks of the parent designs in Table 10 .

\begin{tabular}{rrrrrrr}
\hline$n$ & $R_{1}^{p}$ & $A_{3}$ & $A_{4}$ & \multicolumn{3}{c}{$F A_{3}$} \\
\hline 12 & 1 & 16 & 39 & 0 & 0 & 64 \\
11 & 1 & 12 & 26 & 0 & 0 & 48 \\
10 & 1 & 8 & 18 & 0 & 0 & 32 \\
9 & 11 & 4 & 14 & 0 & 0 & 16 \\
8 & 34 & 0 & 14 & 0 & 0 & 0 \\
7 & 64 & 0 & 7 & 0 & 0 & 0 \\
6 & 1 & 0 & 3 & 0 & 0 & 0 \\
& 46 & 0 & 3 & 0 & 0 & 0 \\
& 71 & 0 & 3 & 0 & 0 & 0 \\
5 & 1 & 0 & 1 & 0 & 0 & 0 \\
& 6 & 0 & 0 & 0 & 0 & 0 \\
& 50 & 0 & 1 & 0 & 0 & 0 \\
4 & 1 & 0 & 0 & 0 & 0 & 0 \\
3 & 1 & 0 & 0 & 0 & 0 & 0 \\
\hline
\end{tabular}


however, not received any attention in the literature. The optimal blocking arrangements we obtained are given in Tables 12-14.

Table 12 shows the best blocking arrangements obtained from designs of the type OA $\left(18 ; 3^{n} \times 2\right)$. These designs can be used to arrange $\mathrm{OA}\left(18 ; 3^{n}\right)$ designs in two blocks and to arrange $\mathrm{OA}\left(18 ; 3^{n-1} \times 2\right)$ designs in three blocks. Remarkably, the parent designs that give the best arrangements in two blocks are different from the parent designs that give the best arrangements in three blocks in all but one case. Another noteworthy result is that Taguchi's $L_{18}$ array, which is the design with $n=7$ and $R_{1}=3$ in Table 12 , cannot be used to obtain an optimal arrangement in two blocks. The child designs of the optimal arrangements in two blocks are the three-level designs with $R_{1}=1$ in Table 13. Table 12 shows that the corresponding parent designs also have $R_{1}=1$ for $n=7$ and 6 , but, for $n=5$ and $4, R_{1}$ equals 5 and 4 , respectively. Finally, the optimal arrangement in three blocks derived from a parent design with $n=3$ has a full factorial $3^{2} \times 2$ child design. There is no extensive confounding of the two-factor interactions with the blocks for this arrangement. Since two degrees of freedom are available for estimating the residual error variance in case a model including two-factor interactions is estimated, we do not recommend using the $W_{1}^{-}$- or $W_{2}^{-}$-criterion in this scenario.

Except for the case where $n=7$ and $R_{1}=1$ and the case where $n=4$ and $R_{1}=4$, all the designs in Table 12 can be derived from Taguchi's $L_{18}$ array by dropping one or more columns.

Optimal arrangements of pure three-level 18-run designs in three blocks of size six are given in Table 13. For all designs, the first column is used for the blocking factor. The child designs of all optimal blocking

Table 12: Optimal blocking arrangements based on parent designs of the type OA $\left(18 ; 3^{n} \times 2\right)$. Elements of the $F A_{3}$ vector correspond to $A_{3}$ values of $2,1,2 / 3,1 / 2$, and $4 / 9$. An 'x' indicates $W_{1}$ - and $W_{2}$-optimal arrangements; a ' $y$ ' indicates $W_{1}^{-}$- and $W_{2}^{-}$-optimal arrangements; a ' $z$ ' indicates $W_{3}$-optimal arrangements.

\begin{tabular}{|c|c|c|c|c|c|c|c|c|c|c|c|c|}
\hline$n$ & ID & $R_{1}$ & $R_{2}$ & $A_{3}$ & $A_{4}$ & & & $F A$ & & & 2 blocks & 3 blocks \\
\hline \multirow[t]{2}{*}{7} & 1 & 1 & 1 & 28 & 52.5 & 1 & 0 & 21 & 16 & 9 & 1:1;xyz & \\
\hline & 2 & 3 & 3 & 28 & 52.5 & 1 & 6 & 9 & 28 & 0 & & 1:1;xyz \\
\hline \multirow[t]{3}{*}{6} & 1 & 1 & 1 & 16 & 28.5 & 0 & 0 & 9 & 20 & 0 & $1: 1 ; x y z$ & \\
\hline & 2 & 3 & 3 & 16.67 & 25.83 & 0 & 0 & 15 & 8 & 6 & & $1: 3 ; z$ \\
\hline & 3 & 5 & 5 & 17 & 24.5 & 0 & 6 & 6 & 14 & 0 & & $3: 1 ; x y$ \\
\hline \multirow[t]{5}{*}{5} & 1 & 1 & 11 & 8.5 & 12 & 0 & 3 & 3 & 7 & 0 & & $5: 1 ; \mathrm{x}$ \\
\hline & 2 & 3 & 1 & 8.67 & 11.5 & 0 & 0 & 6 & 4 & 6 & & \\
\hline & 3 & 4 & 2 & 8.78 & 11.17 & 0 & 0 & 6 & 6 & 4 & & $1: 4 ; z$ \\
\hline & 4 & 5 & 3 & 9 & 10.5 & 0 & 0 & 6 & 10 & 0 & 1:1;xyz & \\
\hline & 5 & 19 & 13 & 10 & 7.5 & 0 & 6 & 3 & 4 & 0 & & $3: 1 ; y$ \\
\hline \multirow[t]{4}{*}{4} & 1 & 1 & 33 & 3.5 & 4.5 & 0 & 3 & 0 & 1 & 0 & & $1: 1 ; \mathrm{xz}$ \\
\hline & 2 & 4 & 1 & 3.78 & 3.94 & 0 & 0 & 0 & 4 & 4 & $1: 1 ; \mathrm{xz}$ & \\
\hline & 3 & 10 & 8 & 4 & 3.5 & 0 & 0 & 3 & 2 & 2 & & $1: 1 ; \mathrm{y}$ \\
\hline & 4 & 26 & 12 & 4.67 & 2.17 & 0 & 0 & 4 & 4 & 0 & $1: 1 ; \mathrm{y}$ & \\
\hline \multirow[t]{2}{*}{3} & 1 & 1 & 1 & 0.5 & 1.5 & 0 & 0 & 0 & 1 & 0 & $1: 1 ; \mathrm{xz}$ & $1: 1 ; x z$ \\
\hline & 2 & 10 & 8 & 1.83 & 0.17 & 0 & 0 & 2 & 1 & 0 & $1: 1 ; \mathrm{y}$ & \\
\hline
\end{tabular}


arrangements have $R_{1}=R_{2}=1$. The seven-factor design with $R_{1}=R_{2}=1$ in Table 13 does not yield any optimal blocking pattern, but it is included in the table because it is the child design of the first optimal blocking arrangement involving two blocks in Table 12.

Finally, Table 14 gives optimal blocking arrangements of $\mathrm{OA}\left(18 ; 3^{n}\right)$ designs in six blocks of size three and of $\mathrm{OA}\left(18 ; 6 \times 3^{n-1}\right)$ designs in three blocks of size six. Dropping any three-level column of any design in Table 14 results in the same projection, so that every possible arrangement in three blocks is optimal with respect to each of our five criteria. As there is only one six-level factor, using that factor as a blocking factor yields designs that are optimal with respect to each of our five criteria as well.

\subsection{Designs with 20 runs}

In this section, we discuss optimal blocking arrangements for 20-run designs. Optimal blocking arrangements of pure two-level OA $\left(20 ; 2^{n}\right)$ designs in two blocks of size ten are given by CLY. In Table 15, we present optimal arrangements of $\mathrm{OA}\left(20 ; 2^{n}\right)$ designs in five blocks of size four, and optimal arrangements of $\mathrm{OA}(20 ; 5 \times$ $2^{n}$ ) designs in two blocks of size ten. In Table 16, we give the $A_{3}$ and $A_{4}$ values and the $F A_{3}$ vector of

Table 13: Optimal blocking arrangements based on parent designs of the type $\mathrm{OA}\left(18 ; 3^{n}\right)$. Elements of the $F A_{3}$ vector correspond to $A_{3}$ values of $2,1,2 / 3$, and $1 / 2$. An ' $\mathrm{x}$ ' indicates $W_{1}$ - and $W_{2}$-optimal arrangements; a ' $y$ ' indicates $W_{1}^{-}$- and $W_{2}^{-}$-optimal arrangements; a ' $z$ ' indicates $W_{3}$-optimal arrangements.

\begin{tabular}{|c|c|c|c|c|c|c|c|c|c|}
\hline \multirow{3}{*}{$\frac{n}{7}$} & ID & $R_{1}, R_{2}$ & $A_{3}$ & $A_{4}$ & \multicolumn{4}{|c|}{$F A_{3}$} & 3 blocks \\
\hline & 1 & 1 & 22.0 & 34.5 & 1 & 0 & 18 & 16 & \\
\hline & 2 & 3 & 22.0 & 34.5 & 1 & 6 & 0 & 28 & 1:1;xyz \\
\hline \multirow[t]{2}{*}{6} & 1 & 1 & 10.0 & 22.5 & 0 & 0 & 0 & 20 & 1:1;xz \\
\hline & 2 & 8 & 13.0 & 13.5 & 1 & 3 & 0 & 16 & $1: 1 ; \mathrm{y}$ \\
\hline \multirow[t]{2}{*}{5} & 1 & 1 & 5.0 & 7.5 & 0 & 0 & 0 & 10 & 1:1;xz \\
\hline & 2 & 10 & 8.0 & 1.5 & 0 & 6 & 0 & 4 & $1: 1 ; \mathrm{y}$ \\
\hline \multirow[t]{2}{*}{4} & 1 & 1 & 2.0 & 1.5 & 0 & 0 & 0 & 4 & $1: 1 ; \mathrm{xz}$ \\
\hline & 2 & 9 & 3.5 & 0.0 & 1 & 0 & 0 & 3 & $1: 1 ; y$ \\
\hline 3 & 1 & 1 & 0.5 & 0.0 & 0 & 0 & 0 & 1 & $1: 1 ; x y z$ \\
\hline
\end{tabular}

Table 14: Optimal blocking arrangements based on parent designs of the type $\mathrm{OA}\left(18 ; 6 \times 3^{n}\right)$. Elements of the $F A_{3}$ vector correspond to $A_{3}$ values of 2 and $1 / 2$. An 'x' indicates $W_{1}$ - and $W_{2}$-optimal arrangements; a 'y' indicates $W_{1}^{-}$- and $W_{2}^{-}$-optimal arrangements; a 'z' indicates $W_{3}$-optimal arrangements.

\begin{tabular}{rrrrrrcc}
\hline$n$ & $R_{1}$ & $A_{3}$ & $A_{4}$ & \multicolumn{2}{c}{$F A_{3}$} & 3 blocks & 6 blocks \\
\hline 6 & 1 & 40.0 & 52.5 & 15 & 20 & $1: 1 ; \mathrm{xyz}$ & $1: 1 ; \mathrm{xyz}$ \\
5 & 1 & 25.0 & 22.5 & 10 & 10 & $1: 1 ; \mathrm{xyz}$ & $1: 1 ; \mathrm{xyz}$ \\
4 & 1 & 14.0 & 7.5 & 6 & 4 & $1: 1 ; \mathrm{xyz}$ & $1: 1 ; \mathrm{xyz}$ \\
3 & 1 & 6.5 & 1.5 & 2 & 2 & $1: 1 ; \mathrm{xyz}$ & $1: 1 ; \mathrm{xyz}$ \\
2 & 1 & 2.0 & - & 1 & 0 & $1: 1 ; \mathrm{xyz}$ & $1: 1 ; \mathrm{xyz}$ \\
\hline
\end{tabular}


the two-level child designs obtained by dropping the five-level column from the parent designs that give the optimal arrangements in blocks of size four.

Table 15 shows one case where the $W_{1}^{-}$-optimal blocking arrangement differs from the $W_{2}^{-}$-optimal one. For six two-level factors $(n=6)$ and five blocks, the design with $R_{1}=3$ allows a blocking arrangement with $A_{3}^{c}, A_{4}^{c}, A_{21}$ and $A_{31}$ values of $0.8,0.6,4.8-0.8=4$ and $5.8-0.6=5.2$, respectively, while the design ranked ninth allows an arrangement with $A_{3}^{c}, A_{4}^{c}, A_{21}$ and $A_{31}$ values of $0.8,0.92,5.2-0.8=4.4$ and $4.92-0.92=4$, respectively. The former blocking arrangement is $W_{1}^{-}$-optimal (because it has a smaller $A_{4}^{c}$ value), whereas the latter is $W_{2}^{-}$-optimal (because it has a larger $A_{21}$ value).

Table 15: Optimal blocking arrangements based on parent designs of the type OA $\left(20 ; 5 \times 2^{n}\right)$. Elements of the $F A_{3}$ vector correspond to $A_{3}$ values of $1,4 / 5,2 / 5,9 / 25$, and $1 / 25$. An 'x' indicates $W_{1^{-}}$and $W_{2}$ optimal arrangements; a ' $y$ ' indicates $W_{1}^{-}$- and $W_{2}^{-}$-optimal arrangements; a ' $\mathrm{y}_{1}$ ' indicates $W_{1}^{-}$-optimal arrangements; a ' $\mathrm{y}_{2}$ ' indicates $W_{2}^{-}$-optimal arrangements; a ' $z$ ' indicates $W_{3}$-optimal arrangements.

\begin{tabular}{rrrrrrrrrrrcc}
\hline$n$ & ID & $R_{1}$ & $R_{2}$ & $A_{3}$ & $A_{4}$ & & & $F A_{3}$ & & 2 blocks & 5 blocks \\
\hline 8 & 1 & 1 & 1 & 10.88 & 16.24 & 0 & 0 & 20 & 2 & 54 & $1: 1 ; \mathrm{xyz}$ & $\mathrm{xyz}$ \\
7 & 1 & 1 & 1 & 7.72 & 9.64 & 0 & 0 & 15 & 1 & 34 & $2: 6 ; \mathrm{xz}$ & $\mathrm{xyz}$ \\
& 2 & 2 & 2 & 8.04 & 9.32 & 0 & 0 & 15 & 2 & 33 & $1: 6 ; \mathrm{y}$ & \\
6 & 1 & 1 & 1 & 4.72 & 6.2 & 0 & 0 & 9 & 1 & 19 & & \\
& 2 & 3 & 5 & 4.8 & 5.8 & 0 & 0 & 10 & 0 & 20 & $1: 1 ; \mathrm{xyz}$ & $\mathrm{xy}_{1} \mathrm{z}$ \\
& 3 & 6 & 5 & 5.12 & 5.4 & 0 & 0 & 10 & 1 & 19 & & \\
& 4 & 9 & 12 & 5.2 & 4.92 & 0 & 0 & 11 & 0 & 20 & & $\mathrm{y}_{2}$ \\
5 & 1 & 1 & 1 & 2.4 & 3.8 & 0 & 0 & 5 & 0 & 10 & $1: 1 ; \mathrm{xz}$ & $\mathrm{xz}$ \\
& 2 & 9 & 10 & 3.2 & 2.6 & 0 & 0 & 7 & 0 & 10 & $1: 1 ; \mathrm{y}$ & \\
& 3 & 16 & 18 & 3.6 & 1.8 & 0 & 2 & 4 & 0 & 10 & & $\mathrm{y}$ \\
4 & 1 & 1 & 1 & 1.36 & 1.48 & 0 & 0 & 3 & 0 & 4 & $1: 3 ; \mathrm{xz}$ & $\mathrm{xz}$ \\
& 2 & 9 & 5 & 1.76 & 1.08 & 0 & 0 & 4 & 0 & 4 & $1: 3 ; \mathrm{y}$ & \\
3 & 1 & 1 & 1 & 0.04 & 0.96 & 0 & 0 & 0 & 0 & 1 & $1: 1 ; \mathrm{xz}$ & $\mathrm{xz}$ \\
& 2 & 3 & 3 & 0.44 & 0.56 & 0 & 0 & 1 & 0 & 1 & & \\
2 & 1 & 1 & 1 & 0 & - & 0 & 0 & 0 & 0 & 0 & $1: 1 ; \mathrm{xz}$ & $\mathrm{xz}$ \\
\hline
\end{tabular}

Table 16: Properties of two-level child designs obtained from parent of designs of the type OA $\left(20 ; 5 \times 2^{n}\right)$. Elements of the $F A_{3}$ vector correspond to $A_{3}$ values of $1,4 / 5,2 / 5,9 / 25$, and $1 / 25$. The column labeled $R_{1}^{p}$ gives the $R_{1}$ ranks of the parent designs in Table 15

\begin{tabular}{rrrrrrrrr}
\hline$n$ & $R_{1}^{p}$ & $A_{3}$ & $A_{4}$ & \multicolumn{5}{c}{$F A_{3}$} \\
\hline 8 & 1 & 2.88 & 4.72 & 0 & 0 & 0 & 2 & 54 \\
7 & 1 & 1.72 & 2.36 & 0 & 0 & 0 & 1 & 34 \\
6 & 3 & 0.80 & 0.60 & 0 & 0 & 0 & 0 & 20 \\
& 9 & 0.80 & 0.92 & 0 & 0 & 0 & 0 & 20 \\
5 & 1 & 0.40 & 0.20 & 0 & 0 & 0 & 0 & 10 \\
& 16 & 0.40 & 0.20 & 0 & 0 & 0 & 0 & 10 \\
4 & 1 & 0.16 & 0.04 & 0 & 0 & 0 & 0 & 4 \\
3 & 1 & 0.04 & - & 0 & 0 & 0 & 0 & 1 \\
\hline
\end{tabular}


Finally, we neither give $W_{1}^{-}$-optimal or $W_{2}^{-}$-optimal five-block arrangements for up to four two-level factors, nor $W_{1}^{-}$-optimal or $W_{2}^{-}$-optimal two-block arrangements with up to two two-level factors and one five-level factor, as it is possible to fit a model with all main effects and two-factor interaction effects in these cases.

\subsection{Designs with 27 runs}

Three-level designs with 27 runs can be used to study the effects of up to 13 factors. There are 68 nonisomorphic 13-factor designs in 27 runs (Lam and Tonchev, 1996). Their projective properties in the absence of blocking have been extensively studied by Xu et al. (2004). To find the optimal blocking arrangements, we started from a complete enumeration of all non-isomorphic designs for four up to 13 factors. While evaluating these designs, we encountered several 27-run designs that have better $F A_{3}$ vectors than the designs listed in Xu et al. (2004). We discuss this in detail in Appendix B. In this section, we focus on the best blocking arrangements.

Table 17 lists the optimal blocking arrangements for 27-run three-level designs in three blocks of size nine. One remarkable result is that, in five cases, three different parent designs are needed to cover the five blocking optimality criteria. Another remarkable result is that the child designs of the $W_{1^{-}}, W_{2^{-}}, W_{1}^{-}$- and

$W_{2}^{-}$-optimal blocking arrangements for $n=6$ have $R_{1}=4$ only. This clearly demonstrates that starting from the best child design does not guarantee finding an optimal blocking arrangement.

We obtained similar results for the $W_{3}$-optimal blocking arrangements of ten-factor, eight-factor and seven-factor child designs. The child design of the $W_{3}$-optimal arrangement obtained from the 11 -factor parent design with $R_{1}=R_{3}=3$ is only 275 th in the $R_{1}$ ranking and only 5 th in the $R_{2}$ ranking of all ten-factor designs. This is due to the fact that none of the ten-factor designs with a better $R_{1}$ or $R_{2}$ ranking can be obtained by projection from an 11-factor design. Therefore, they cannot be arranged in blocks so that the main effects can be estimated independently from the block effects.

The eight-factor child design corresponding to the $W_{3}$-optimal blocking arrangement obtained from the nine-factor parent design with $R_{1}=2645$ and $R_{2}=1$ is 5259 th in the $R_{1}$ ranking and third in the $R_{2}$ ranking of all eight-factor designs. Neither the first nor the second design in the $R_{2}$ ranking of all eightfactor designs can be obtained from a nine-factor parent design by projection. Finally, the seven-factor child design corresponding to the $W_{3}$-optimal blocking arrangement obtained from the eight-factor parent design with $R_{1}=7153$ and $R_{2}=360$ is $2903 \mathrm{rd}$ in the $R_{1}$ ranking and 89 th in the $R_{2}$ ranking of all seven-factor designs. None of the seven-factor designs with a better $R_{1}$ or $R_{2}$ ranking can be obtained by projection from an eight-factor design, and, hence, no desirable blocking arrangement exists for them.

In all other cases, the child designs corresponding to optimal blocking arrangements are listed first in 
the $R_{1}$ or $R_{2}$ rankings. So, in many cases, it is possible to find desirable blocking arrangements for the best possible child designs.

Table 18 lists the orthogonal blocking arrangements obtained from designs of the type $\mathrm{OA}\left(27 ; 9 \times 3^{n}\right)$. These designs allow us to arrange $\mathrm{OA}\left(27 ; 3^{n}\right)$ designs in nine blocks of size three, and to arrange $\mathrm{OA}(27 ; 9 \times$ $3^{n-1}$ ) designs in three blocks of size nine. For the arrangements in three blocks of size nine, the characteristics of the child designs are listed in Table 18 too. For the arrangements in nine blocks of size three, the child

Table 17: Optimal blocking arrangements based on parent designs of the type $\mathrm{OA}\left(27 ; 3^{n}\right)$. Elements of the $F A_{3}$ vector correspond to $A_{3}$ values of $2,20 / 27,2 / 3,14 / 27,4 / 9$, and $8 / 27$. An ' $\mathrm{x}$ ' indicates $W_{1}$ - and $W_{2}$-optimal arrangements; a ' $\mathrm{y}$ ' indicates $W_{1}^{-}$- and $W_{2}^{-}$-optimal arrangements; a 'z' indicates $W_{3}$-optimal arrangements.

\begin{tabular}{|c|c|c|c|c|c|c|c|c|c|c|c|c|}
\hline$n$ & ID & $R_{1}$ & $R_{2}$ & $A_{3}$ & $A_{4}$ & \multicolumn{6}{|c|}{$F A_{3}$} & 3 blocks \\
\hline 13 & 1 & 1 & 1 & 104 & 468 & 0 & 0 & 52 & 0 & 156 & 0 & $1: 1 ; x y z$ \\
\hline 12 & 1 & 1 & 1 & 80 & 324 & 0 & 0 & 40 & 0 & 120 & 0 & $1: 1 ; x y z$ \\
\hline \multirow[t]{2}{*}{11} & 1 & 1 & 1 & 60 & 216 & 0 & 0 & 30 & 0 & 90 & 0 & $1: 275 ; z$ \\
\hline & 2 & 78 & 78 & 60 & 216 & 2 & 0 & 40 & 0 & 60 & 9 & $7: 1 ; x y$ \\
\hline \multirow[t]{4}{*}{10} & 1 & 1 & 607 & 42 & 144 & 2 & 0 & 33 & 0 & 36 & 0 & \\
\hline & 2 & 6 & 1272 & 42 & 144 & 3 & 0 & 36 & 18 & 0 & 9 & $4: 1 ; x y$ \\
\hline & 3 & 275 & 5 & 43.33 & 138.67 & 0 & 0 & 21 & 0 & 66 & 0 & \\
\hline & 4 & 1214 & 1 & 44 & 136 & 0 & 0 & 3 & 27 & 27 & 54 & 1:2645;z \\
\hline \multirow[t]{3}{*}{9} & 1 & 1 & 4885 & 24 & 108 & 3 & 0 & 27 & 0 & 0 & 0 & $1: 1 ; \mathrm{x}$ \\
\hline & 2 & 680 & 4003 & 30 & 84 & 2 & 0 & 25 & 14 & 0 & 7 & $4: 1 ; \mathrm{y}$ \\
\hline & 3 & 2645 & 1 & 30.44 & 82.67 & 0 & 0 & 2 & 18 & 21 & 36 & 1:5259;z \\
\hline \multirow[t]{5}{*}{8} & 1 & 1 & 13835 & 16 & 60 & 2 & 0 & 18 & 0 & 0 & 0 & $1: 1 ; \mathrm{x}$ \\
\hline & 2 & 2530 & 1 & 19.85 & 47.41 & 0 & 0 & 0 & 16 & 12 & 21 & \\
\hline & 3 & 5259 & 3 & 20.3 & 45.85 & 0 & 0 & 1 & 11 & 16 & 23 & \\
\hline & 4 & 7153 & 360 & 20.52 & 45.41 & 0 & 1 & 1 & 12 & 15 & 21 & 1:2903;z \\
\hline & 5 & 14783 & 14394 & 22 & 42 & 3 & 0 & 24 & 0 & 0 & 0 & $1: 1 ; y$ \\
\hline \multirow{6}{*}{7} & 1 & 1 & 71407 & 10 & 30 & 1 & 0 & 12 & 0 & 0 & 0 & \\
\hline & 2 & 3 & 77742 & 10 & 30 & 2 & 0 & 9 & 0 & 0 & 0 & $5: 1 ; \mathrm{x}$ \\
\hline & 3 & 2903 & 89 & 12 & 24.44 & 0 & 0 & 0 & 10 & 15 & 6 & \\
\hline & 4 & 7745 & 7 & 12.3 & 23.93 & 0 & 0 & 0 & 4 & 7 & 24 & 1:1944;z \\
\hline & 5 & 8841 & 1 & 12.3 & 25.04 & 0 & 0 & 0 & 0 & 15 & 19 & \\
\hline & 6 & 78098 & 78908 & 16 & 18 & 8 & 0 & 0 & 0 & 0 & 0 & $1: 1 ; y$ \\
\hline \multirow[t]{5}{*}{6} & 1 & 1 & 62510 & 4 & 18 & 2 & 0 & 0 & 0 & 0 & 0 & $1: 4 ; \mathrm{x}$ \\
\hline & 2 & 7 & 15037 & 5.33 & 14 & 0 & 0 & 8 & 0 & 0 & 0 & \\
\hline & 3 & 599 & 67 & 10 & 6 & 0 & 0 & 0 & 1 & 4 & 13 & $4: 42 ; \mathrm{z}$ \\
\hline & 4 & 1944 & 1 & 6.15 & 11.78 & 0 & 0 & 0 & 0 & 3 & 17 & \\
\hline & 5 & 62548 & 62550 & 6.37 & 11.56 & 5 & 0 & 0 & 0 & 0 & 0 & $1: 4 ; y$ \\
\hline \multirow[t]{7}{*}{5} & 1 & 1 & 849 & 2 & 6 & 0 & 0 & 1 & 0 & 3 & 0 & \\
\hline & 2 & 2 & 2110 & 2 & 6 & 0 & 0 & 3 & 0 & 0 & 0 & $1: 1 ; \mathrm{x}$ \\
\hline & 3 & 4 & 6060 & 2 & 6 & 1 & 0 & 0 & 0 & 0 & 0 & \\
\hline & 4 & 42 & 1 & 2.67 & 4.67 & 0 & 0 & 0 & 0 & 0 & 9 & \\
\hline & 5 & 44 & 66 & 2.67 & 4.67 & 0 & 0 & 0 & 0 & 6 & 0 & $1: 1 ; \mathrm{z}$ \\
\hline & 6 & 49 & 2362 & 2.67 & 4.67 & 0 & 0 & 4 & 0 & 0 & 0 & \\
\hline & 7 & 2591 & 6111 & 4 & 2 & 2 & 0 & 0 & 0 & 0 & 0 & 1:1;y \\
\hline 4 & 1 & 1 & 1 & 0 & 2 & 0 & 0 & 0 & 0 & 0 & 0 & $1: 1 ; \mathrm{xz}$ \\
\hline
\end{tabular}


designs' $A_{3}$ and $A_{4}$ values and their $F A_{3}$ vector are listed in Table 17.

All but one $W_{1^{-}}, W_{2^{-}}, W_{1}^{-}$- and $W_{2}^{-}$-optimal blocking arrangements have child designs with $R_{1}=1$. The only exception is for $n=5$ and nine blocks. The $3^{5}$ child design for that case has an $F A_{3}$ vector of $(1,0,0,0,0,0)$, while the best 5 -factor design in the $R_{1}$ ranking has an $F A_{3}$ vector of $(0,0,1,0,3,0)$. Note from the list of six-factor designs in Table 17 that the latter design cannot be blocked orthogonally in three blocks either.

Most $W_{3}$-optimal blocking arrangements in Table 18 have child designs with $R_{2}=1$. The two exceptions are the $W_{3}$-optimal arrangements for six and five three-level factors and nine blocks. The six-factor child design has $R_{2}=15037$, and the five-factor child design has $R_{2}=2362$. Clearly, there is a high price to be paid here for orthogonal blocking in nine blocks in terms of the $R_{2}$ ranking of the child designs.

\section{Discussion}

In this paper, we proposed a simple method to arrange orthogonal designs of resolution 3 or strength 2 in blocks. The starting point of our method is an exhaustive catalog of non-isomorphic parent designs. We then use single replacement of a column in the designs by the blocking factor.

The method works well even when the catalogs have tens of thousands of arrays. However, it is computationally infeasible for cases involving even larger numbers of arrays. For instance, for 24 runs, there are 52,912,678 non-isomorphic designs for 11 two-level factors and 51,154,497 non-isomorphic designs for

Table 18: Optimal blocking arrangements based on parent designs of the type $\mathrm{OA}\left(27 ; 9 \times 3^{n}\right)$. Elements of the $F A_{3}$ vector correspond to $A_{3}$ values of 2 and $2 / 3$. An 'x' indicates $W_{1^{-}}$and $W_{2}$-optimal arrangements; a ' $y$ ' indicates $W_{1}^{-}$- and $W_{2}^{-}$-optimal arrangements; a ' $z$ ' indicates $W_{3}$-optimal arrangements.

\begin{tabular}{rrrrrrrrrr}
\hline$n$ & ID & $R_{1}$ & $R_{2}$ & $A_{3}$ & $A_{4}$ & \multicolumn{1}{c}{$F A_{3}$} & 3 blocks & 9 blocks \\
\hline 9 & 1 & 1 & 1 & 96 & 252 & 39 & 27 & $1: 1 ; \mathrm{xyz}$ & $1: 1 ; \mathrm{xyz}$ \\
8 & 1 & 1 & 1 & 72 & 156 & 30 & 18 & $1: 1 ; \mathrm{xyz}$ & $1: 1 ; \mathrm{xyz}$ \\
7 & 1 & 1 & 1 & 52 & 90 & 22 & 12 & $1: 3 ; \mathrm{z}$ & $1: 1 ; \mathrm{xyz}$ \\
& 2 & 3 & 3 & 52 & 90 & 23 & 9 & $7: 1 ; \mathrm{xy}$ & \\
6 & 1 & 1 & 6 & 34 & 54 & 17 & 0 & $1: 1 ; \mathrm{x}$ & $1: 1 ; \mathrm{xy}$ \\
& 2 & 3 & 1 & 35.33 & 48.67 & 15 & 8 & $1: 3 ; \mathrm{z}$ & $1: 7 ; \mathrm{z}$ \\
& 3 & 7 & 7 & 36 & 46 & 18 & 0 & $6: 1 ; \mathrm{y}$ & \\
5 & 1 & 1 & 4 & 22 & 24 & 11 & 0 & $1: 1 ; \mathrm{xz}$ & $1: 4 ; \mathrm{xy}$ \\
& 2 & 3 & 1 & 22.67 & 22 & 10 & 4 & & $1: 49 ; \mathrm{z}$ \\
& 3 & 7 & 7 & 24 & 18 & 12 & 0 & $1: 1 ; \mathrm{y}$ & \\
4 & 1 & 1 & 1 & 12 & 10 & 6 & 0 & $1: 1 ; \mathrm{xz}$ & $1: 1 ; \mathrm{xyz}$ \\
& 2 & 5 & 5 & 14 & 6 & 7 & 0 & $1: 1 ; \mathrm{y}$ & \\
3 & 1 & 1 & 1 & 6 & 2 & 3 & 0 & $1: 1 ; \mathrm{xz}$ & $1: 1 ; \mathrm{xyz}$ \\
& 2 & 5 & 5 & 8 & 0 & 4 & 0 & $1: 1 ; \mathrm{y}$ & \\
2 & 1 & 1 & 1 & 2 & - & 1 & 0 & $1: 1 ; \mathrm{xyz}$ & $1: 1 ; \mathrm{xyz}$ \\
\hline
\end{tabular}


12 two-level factors (Schoen et al., 2010). These numbers are too large to identify all optimal blocking arrangements with our method.

Applying our method will also be problematic for finding optimal blocking arrangements of resolution-4 or strength-3 orthogonal designs. This is because it is sufficient for the parent design to have resolution 3 , but the child design must have resolution 4 . For example, to arrange a resolution- 4 design of the type $\mathrm{OA}\left(54 ; 3^{5}\right)$ in three blocks of size 18, we would need a complete catalog of all resolution-3 non-isomorphic parent designs of the type $\mathrm{OA}\left(54 ; 3^{6}\right)$. Not only is generating such a catalog computationally very intensive, but the resolution of every possible child design would have to be determined as well. This is also computationally demanding. As a result, for designs with a resolution of 4 or more, alternative blocking strategies are needed.

\section{Appendix A. Comparison with Cheng et al. (2004)}

We listed the $W_{1^{-}}, W_{2^{-}}, W_{1}^{-}-, W_{2}^{-}$- and $W_{3}$-optimal arrangements of 16-run two-level designs in four blocks of size four in Table 10. In this appendix, we compare our results with those of Cheng et al. (2004, CLY).

\section{A1. The $F A_{3}$ vector of designs of the type $\mathrm{OA}\left(16 ; 4 \times 2^{n}\right)$}

At first sight, there is a discrepancy between our work and that of CLY. This is due to the fact that our method sometimes results in an $F A_{21}$ vector with a non-zero entry for $A_{3}=0.5$, while the possible $A_{3}$ values of three-factor designs are either $1,0.25$, or 0 for pure two-level designs in 16 runs. Therefore, none of the designs in CLY have $A_{3}$ values of 0.5 .

As an example, consider the difference of the $F A_{3}$ vector of the $\mathrm{OA}\left(16 ; 4 \times 2^{n}\right)$ parent design with ID 1 in Table 10 with the corresponding vector of the child design in Table 11 . In our approach, the $F A_{21}$-vector is $(6-0,24-0,64-64)$ or $(6,24,0)$, with elements corresponding to the $A_{3}$ values $1,0.5$ and 0.25 . In the approach of CLY, the same blocked design has an $F A_{21}$-vector of $(6,48)$, with elements corresponding to the $A_{3}$ values 1 and 0.25 . It turns out that CLY's frequency for an $A_{3}$ value of 0.25 is the sum of our frequency for an $A_{3}$ value of 0.25 and twice our frequency for an $A_{3}$ value of 0.5 .

To see this, it is important to realize that we started from a catalog of non-isomorphic orthogonal 16-run designs, involving $n$ two-level factors and one four-level factor. We applied the single replacement method to this catalog of designs. CLY, however, started from a catalog of two-level designs, replacing two two-level factors with a four-level blocking factor.

In our approach, we encountered two types of three-factor projections. The first type involved three two-level factors, whereas the second type involved two two-level factors and the four-level factor. It is the 
Table 19: $A_{3}$ values and $F A_{3}$ vectors of the three possible non-isomorphic projections into two two-level factors and one four-level factor.

\begin{tabular}{cccccccccc}
\hline Projection & & \multicolumn{3}{c}{ This Paper } & & \multicolumn{3}{c}{ CLY } \\
\cline { 3 - 5 } & $A_{3}$ & 1 & 0.5 & 0 & & 1 & 0.25 & 0 \\
\hline (i) & $F A_{3}$ & 0 & 0 & 1 & & 0 & 0 & 4 \\
(ii) & $F A_{3}$ & 0 & 1 & 0 & & 0 & 2 & 2 \\
(iii) & $F A_{3}$ & 1 & 0 & 0 & & 1 & 0 & 3 \\
\hline
\end{tabular}

second type of projection that causes the difference between our approach and that of CLY.

There are three non-isomorphic three-factor projections involving the four-level factor. If we denote the two-level pseudo-factors corresponding to the four-level factor by $a_{1}$ and $a_{2}$ and the ordinary two-level factors by $B$ and $C$, then the three-factor projections are either (i) a full factorial $4 \times 2^{2}$ design, (ii) an eight-run fractional factorial design with defining relation $I=a_{1} B C$ combined with an eight-run fractional factorial design with defining relation $I=a_{2} B C$, and (iii) a duplicated eight-run fractional factorial design with defining relation $I=a_{1} B C$. The $A_{3}$ values of these projections are $0,0.5$, and 1 , respectively.

We illustrate the calculation of these $A_{3}$ values using projection (ii), because this is where the difference between our approach and that of CLY occurs. The $A_{3}$ value is obtained as follows. The sum of the elementwise products of the contrast columns $a_{1}, B$ and $C$ equals 8 because the three-factor interaction contrast vector $a_{1} B C$ is aliased with the intercept in one half of the design and orthogonal to the intercept in the other half of the design. Following the definition of the $A_{3}$ value in $\mathrm{Xu}$ and $\mathrm{Wu}(2001)$, this contributes an amount of $(8 / 16)^{2}=0.25$ to the $A_{3}$ value of the projection. Similarly, the sum of the element-wise products of the contrast columns $a_{2}, B$ and $C$ equals 8 because the three-factor interaction contrast vector $a_{2} B C$ is also aliased with the intercept in one half of the design and orthogonal to the intercept in the other half of the design. This also contributes an amount of $(8 / 16)^{2}=0.25$ to the $A_{3}$ value of the projection. Finally, the element-wise products of the contrast columns $a_{1}, a_{2}$ and $B$ is zero, as is the sum of the elementwise product of the contrast columns $a_{1}, a_{2}$ and $C$. As a result, the total $A_{3}$ value for this projection is $0.25+0.25+0+0=0.5$.

In contrast with our approach, CLY do not sum the four $A_{3}$ values obtained from $a_{1}, a_{2}, B$ and $C$. So, for the projection (ii) into one four-level factor and two two-level factors, they use the four $A_{3}$ values of 0 , 0, 0.25 and 0.25 separately. As a result, they do not have a value of 0.5.

The $F A_{3}$ vectors and $A_{3}$ values for the projection (i) into a full factorial design and the projection (iii) into a duplicated fractional factorial design can be derived in a similar fashion. For each of the projections, we compare our approach with the approach of CLY in Table 19. We conclude that the frequency of the $A_{3}$ value of 0.25 in CLY can be obtained by adding twice our frequency for an $A_{3}$ value of 0.5 to our frequency 
for an $A_{3}$ value of 0.25 . The frequency of the $A_{3}$ value of 1 in our approach matches that in CLY's approach.

\section{A2. Design comparison}

For each of the cases with 12, 9, 8 and 7 two-level factors, Table 10 contains one optimal arrangement in four blocks. In contrast, CLY report two blocking arrangements, because they consider rankings based on the $A_{i}$ values entirely separately from rankings based on the $F A_{i}$ vectors. Their first arrangement in four blocks is best according to their $A_{i}$-based ranking, and their second arrangement is optimal according to both their $A_{i}$-based ranking and their $F A_{i}$-based ranking. Our $R_{1}$ ranking, which forms the basis for selecting $W_{1^{-}}$ and $W_{2}$-optimal designs, uses the $F A_{3}$ vector to distinguish between designs that have the same $A_{3}$ and $A_{4}$ values. Therefore, the 12-, 9-, 8- and 7-factor designs we report correspond to the designs that are optimal according to CLY's $F A_{i}$-based criterion.

For the 11-factor case involving four blocks, our Table 10 again contains a single blocking arrangement, whereas CLY give two designs. Here too, our design corresponds to the second one listed by CLY. We did not identify their first design because we did not use the $F A_{4}$ vector in our work. This is because we found that the different $A_{4}$ values of four-factor projections in designs with factors acting at more than two levels can be very close to each other. Therefore, the practical importance of a classification based on an $F A_{4}$ vector is questionable for these designs. Note that two-level designs, as opposed to multi-level designs, can only have a few clearly distinct $A_{4}$ values, and classification based on $F A_{4}$ is indeed useful.

For the 10-factor case involving four blocks, CLY list three blocking arrangements. Our Table 10 reports their third design only. For the reason why we did not identify their first design, we refer to the 12-, 9-, 8and 7-factor cases. For the reason why we did not identify their second design, we refer to the 11-factor case.

For the case involving six factors, we identified a $W_{1}$ - and $W_{2}$-optimal blocking arrangement, as well as a design that gives a $W_{3}$-optimal blocking arrangement. Both designs were also given by CLY. Compared to CLY, we identify an additional blocking arrangement which is $W_{1}^{-}$- and $W_{2}^{-}$-optimal. The $W_{1}^{-}$- and $W_{2}^{-}$-criteria were not used by CLY, which explains why they did not report that blocking arrangement. Note that, in all cases with more than six factors, the $W_{1^{-}}, W_{2^{-}}, W_{1}^{-}, W_{2}^{-}$- and $W_{3^{-}}$optimal blocking arrangements coincide. This is no longer the case when the number of factors is at most six.

For the case involving five factors, CLY list four blocking arrangements, while we report only three. Only one of our designs is listed by CLY. The first and third blocking arrangement in CLY are both $W_{1^{-}}$ optimal, provided only $A_{i}$ values are used to rank the blocking arrangements. Our approach to identify $W_{1}$-optimal blocking arrangements is based on $A_{3}$ and $A_{4}$ values, and on the $F A_{3}$ vector to break possible ties, and resulted in a single $W_{1}$-optimal blocking arrangement that outperforms those identified by CLY; 
see the discussion of Table 10 in Section 5.2. That blocking arrangement is also optimal in terms of the $W_{1}^{-}$-criterion.

The second and fourth five-factor blocking arrangement given by CLY are $W_{2}$-optimal. The second blocking arrangement is only optimal when the ranking is based only on $A_{i}$ values, while the fourth design is optimal both when using $A_{i}$ values and $F A_{i}$ vectors. Our mixed ranking identified a $W_{2}$-optimal design that corresponds with the fourth CLY design. Note that the $F A_{21}$ and $F A_{31}$ vectors for the second five-factor CLY design are incorrect. The correct $F A_{21}$ vector is $(2,0)$ and the correct $F A_{31}$ vector is $(4,0)$, where the elements correspond to the $A_{3}$ values 1 and 0.25 , respectively, following the notation of CLY.

Finally, our third five-factor blocking arrangement is based on the $W_{2}^{-}$- criterion, which was not considered by CLY.

\section{Appendix B. Comparison with Xu et al. (2004)}

Xu et al. (2004) studied the $A_{3}$ values and the $F A_{3}$ vectors of all projections from the 68 non-isomorphic $\mathrm{OA}\left(27 ; 3^{13}\right)$ designs reported by Lam and Tonchev (1996) as well as of 1000 randomly generated orthogonal designs with 5 to 10 factors, and listed the best ones. In our search for optimal blocking arrangements, we used the complete enumeration of all 27-run non-isomorphic orthogonal designs instead. For this reason, we were able to identify several designs with better properties in the absence of blocking than those listed by Xu et al. (2004).

Our global search confirmed that Xu et al. (2004) found designs with minimum $A_{3}$ values. For each number of factors, $n$, in Table 17, the minimum $A_{3}$ value is given in the row with $R_{1}=1$. For the case of 10 factors, we were able to find several designs that have a better $F A_{3}$ vector than the best design reported by $\mathrm{Xu}$ et al. (2004). Their design has a frequency of three for $A_{3}=2$, a frequency of nine for $A_{3}=30 / 27$ and a zero frequency for $A_{3}=20 / 27$ among all three-factor projections. Two of the 10-factor designs we found with better $F A_{3}$ vectors are shown in Table 17 . The one with $R_{1}=1$ has a frequency of two for $A_{3}=2$, a zero frequency for $A_{3}=30 / 27$ and a frequency of 33 for $A_{3}=20 / 27$. The one with $R_{1}=6$ has a frequency of three for $A_{3}=2$, a zero frequency for $A_{3}=30 / 27$ and a frequency of 36 for $A_{3}=20 / 27$. Note that none of the three-factor projections for any of the designs in Table 17 resulted in an $A_{3}$ value of 30/27. Finally, all the designs with 5-10 factors that are first in the $R_{2}$ ranking in Table 17 have a better $F A_{3}$ vector than the corresponding designs reported by $\mathrm{Xu}$ et al. (2004).

In conclusion, by going through the complete catalog of all non-isomorphic 27-run three-level designs, we found several attractive designs that were hitherto not known. 


\section{References}

Ai, M. and Zhang, R. (2004). Theory of optimal blocking of nonregular factorial designs. Canadian Journal of Statistics, 32:57-72.

Bisgaard, S. (1994). A note on the definition of resolution for blocked $2^{k-p}$ designs. Technometrics, 36:308311.

Box, G. E. P., Hunter, W. G., and Hunter, J. S. (2005). Statistics for experimenters. 2nd edition, New York: Wiley.

Cheng, S. W., Li, W., and Ye, K. Q. (2004). Blocked nonregular two-level factorial designs. Technometrics, $46: 269-279$.

Cheng, S. W. and Wu, C. F. J. (2002). Choice of optimal blocking schemes in two-level and three-level designs. Technometrics, 44:269-277.

Connor, W. S. and Zelen, M. (1959). Fractional factorial experiment designs for factors at three levels. Applied Mathematics Series 54; Washington DC: US Government Printing Office.

Deng, L. Y. and Tang, B. (1999). Generalized resolution and minimum aberration criteria for PlackettBurman and other nonregular factorial designs. Statistica Sinica, 9:1071-1082.

Fries, A. and Hunter, W. G. (1980). Minimum aberration $2^{k-p}$ designs. Technometrics, 22:601-608.

Hedayat, A. S., Sloane, N. J. A., and Stufken, J. (1999). Orthogonal Arrays: Theory and Applications. Springer.

Lam, C. and Tonchev, V. D. (1996). Classification of affine resolvable 2-(27, 9, 4) designs. Journal of Statistical Planning and Inference, 56:187-202.

National Bureau of Standards (1957). Fractional factorial designs for factors at two levels. Applied Mathematics Series 48; Washington DC: US Government Printing Office.

Rao, C. R. (1947). Factorial experiments derivable from combinatorial arrangements of arrays. Journal of the Royal Statistical Society Supplement, 9:128-139.

Schoen, E. D. (2009). All orthogonal arrays with 18 runs. Quality and Reliability Engineering International, 25:467-480.

Schoen, E. D., Eendebak, P. T., and Nguyen, M. V. M. (2010). Complete enumeration of pure-level and mixed-level orthogonal arrays. Journal of Combinatorial Designs, 18:123-140. 
Sitter, R. R., Chen, J., and Feder, M. (1997). Fractional resolution and minimum aberration in blocked $2^{n-k}$ designs. Technometrics, 39:382-390.

Sun, D. X., Wu, C. F. J., and Chen, Y. (1997). Optimal blocking schemes for $2^{n}$ and $2^{n-p}$ designs. Technometrics, 39:298-307.

Tang, B. and Deng, L. Y. (1999). Minimum $\mathrm{G}_{2}$-aberration for nonregular fractional factorial designs. Annals of Statistics, 27:1914-1926.

Wang, J. C. and Wu, C. J. F. (1992). Nearly orthogonal arrays with mixed levels and small runs. Technometrics, 34:409-422.

Wu, C. F. J. and Hamada, M. S. (2009). Experiments: Planning, Analysis, and Parameter Design Optimization. Wiley, New York, NY, USA.

Xu, H., Cheng, S. W., and Wu, C. F. J. (2004). Optimal projective three-level designs for factor screening and interaction detection. Technometrics, 46:280-292.

Xu, H. and Wu, C. F. J. (2001). Generalized minimum aberration for asymmetrical fractional factorial designs. Annals of Statistics, 29:1066-1077. 